\title{
Combined NMR and UV-vis Spectroscopic Studies of Models for the Hydrogen Bond System in the Active Site of Photoactive Yellow Protein: $\mathrm{H}$-Bond Cooperativity and Medium Effects
}

Benjamin Koeppe*, Peter M. Tolstoy, Jing Guo, Gleb S. Denisov, Hans-Heinrich Limbach

1 Syntheses

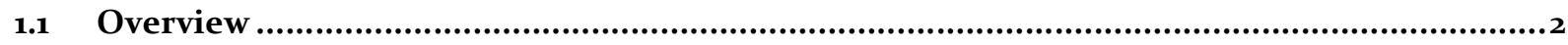

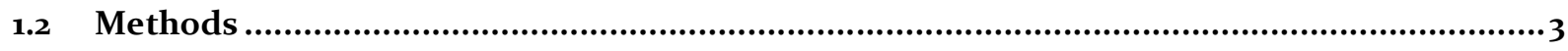

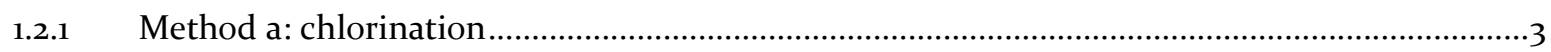

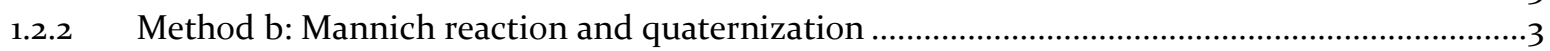

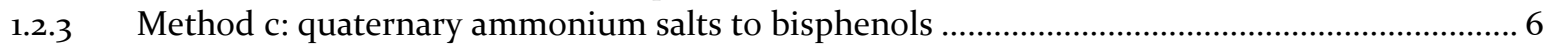

1.2.4 Method d: quaternary ammonium salts to (2-hydroxyphenyl)-acetic acids ..............................7

1.2.5 Method e: reduction to 2-(2-hydroxyphenyl)-ethanol derivatives .......................................... 8

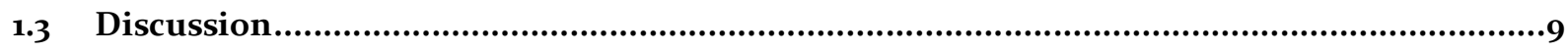

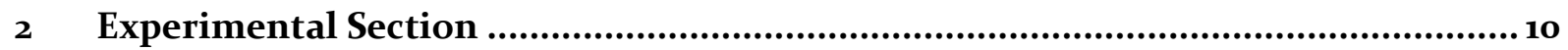

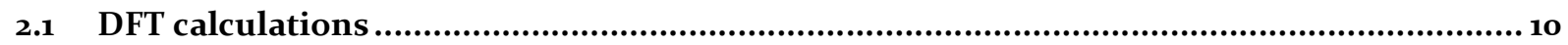

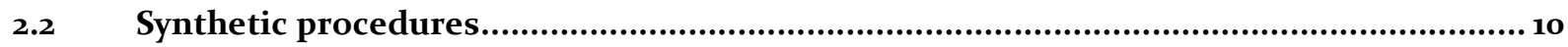

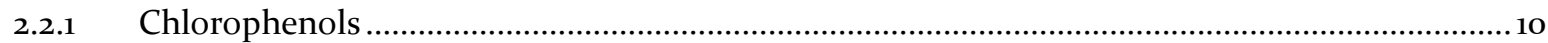

2.2.2 (2-hydroxybenzyl)-trimethylammonium iodides ............................................................... 10

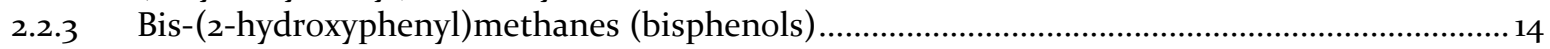

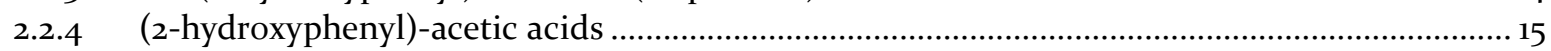

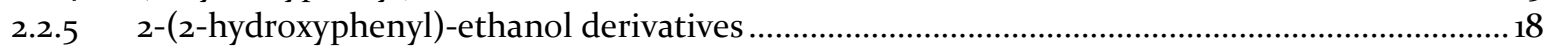

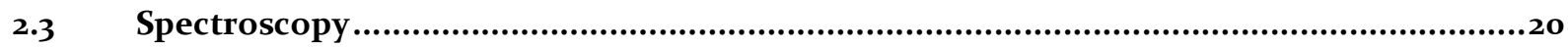

2.3.1 Instrumentation, sample preparation ................................................................................. 20

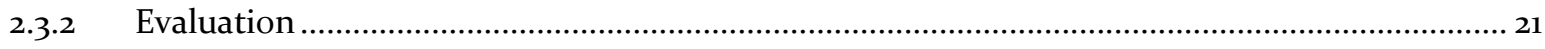

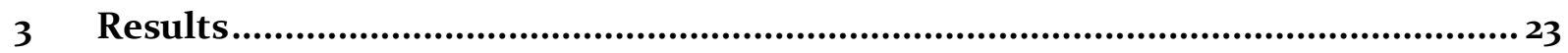

3.1.1 UV-vis absorption spectra of 4-nitrophenols, their anions and homoconjugated anions ........23

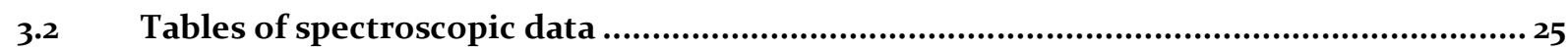

$4 \quad$ References.............................................................................................29 


\section{Syntheses}

\subsection{Overview}

Scheme S 1. Schematic overview of synthetic routes to neutral precursors of mono-anions 4 through $\mathbf{8}$ from simple phenolic compounds employing five types of methods labeled $a-e$ (see main text).<smiles>CC(C)(C)[Si](C)(C)C(=O)c1cc(Cl)cc(Cc2cc(Cl)ccc2O)c1O</smiles>

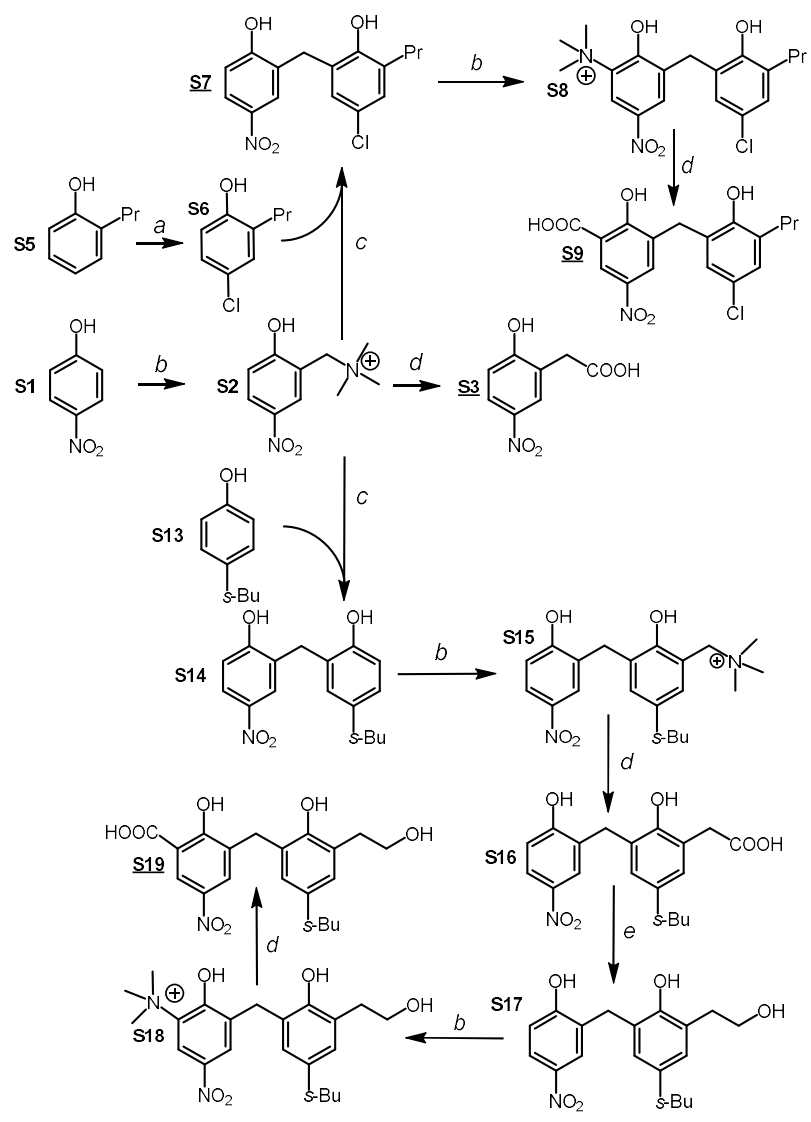

As depicted in Scheme $S_{1}$, neutral precursors of species $\mathbf{1 b}, \mathbf{2 b}, \mathbf{3} \mathbf{b}, \mathbf{3} \mathbf{c}, \mathbf{4} \mathbf{b}\left(\mathbf{S}_{\mathbf{3}}, \mathbf{S}_{\mathbf{7}}, \mathbf{S}_{\mathbf{9}}, \mathbf{S}_{\mathbf{1 2}}\right.$, and $\mathbf{S} \mathbf{1 9}$, respectively, numbers underlined in the scheme) were obtained from commercially available phenols by a combination of five types of conversions $a, b, c, d$ and $e$. Conversion $a$ is single step chlorination. Conversion $b$ is the Mannich reaction of a phenol with formalin and dimethylamine to form the corresponding Mannich 
bases and subsequent quaternization with methly iodide. ${ }^{1}$ The iodides thus obtained were either coupled to another phenol resulting in bis-(2-hydroxyphenyl)-methane derivatives (bisphenols, conversion $c)^{2,3}$ or reacted with cyanide and converted to (2-hydroxyphenyl)-acetic acids through hydrolysis of the resulting nitrile (conversion $d$ ). ${ }^{1}$ Conversion $e$ is a selective reduction of carboxylic groups to hydroxyl groups by borane. $^{4}$

The Freonic solvent mixture $\mathrm{CDF}_{2} \mathrm{Cl} / \mathrm{CDF}_{3}$ was prepared from $\mathrm{CDCl}_{3}$ according to Ref. 5 .

\subsection{Methods}

\subsubsection{Method a: chlorination}

4-chloro-2-n-propylphenol was obtained by chlorination of 2-n-propylphenol with $\mathrm{N}$-chlorosuccinimide (NCS):

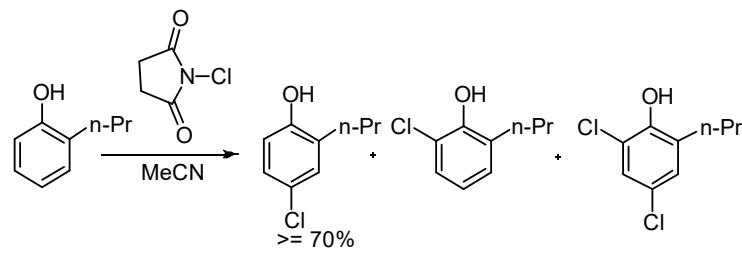

This procedure, given in Section 2.2.1.1, was chosen in analogy to a procedure for bromination by $N$ bromosuccinimide (NBS) described in Ref. 6. It has conveniently been used in several other conversions (Ref. 7). The reaction of phenols with NCS in acetonitrile solution at room or slightly elevated temperatures seems to be generally slower but more selective than that with NBS. However, one should be aware that in the halogenation of phenols by any $N$-halogenosuccinimide, problems in the separation of succinimide from halogenation products can be an issue that should not be underestimated, especially on large scale reactions.

\subsubsection{Method b: Mannich reaction and quaternization}

Compounds listed in Table $S_{1}$ were obtained according to the scheme in Figure $\mathrm{S} 1$; specific procedure can be found in the given subsection to $\mathbf{2 . 2 . 2}$

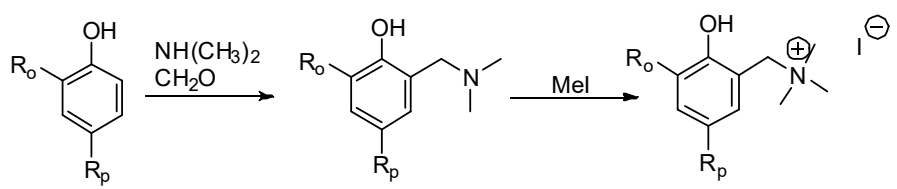

Figure S 1. Mannich reaction of phenolic species to Mannich bases and methylation of Mannich bases to the corresponding tetraalkylammonium iodides. Specific compounds with substituents $R_{p}$ and $R_{o}$ are listed in Table $S$. 
Table $S$ 1. Nomenclature of compounds with substituents $R_{p}$ and $R_{o}$ in Figure $S 1$ and references to experimental procedures.

\begin{tabular}{|c|c|c|c|c|}
\hline $\mathrm{R}_{\mathrm{p}}$ & $\mathrm{R}_{\mathrm{o}}$ & reactant & product & procedure \\
\hline $\mathrm{NO}_{2}$ & $\mathrm{H}$ & S1 & $S_{2}$ & 2.2.2.1 \\
\hline $\mathrm{Cl}$ & & S10 & S11 & 2.2 .2 .2 \\
\hline $\mathrm{NO}_{2}$ & & $S_{7}$ & S8 & 2.2 .2 .3 \\
\hline $\mathrm{s}-\mathrm{Bu}$ & & $\mathrm{S}_{14}$ & $\mathrm{~S}_{15}$ & 2.2 .2 .4 \\
\hline $\mathrm{NO}_{2}$ & & $\mathrm{~S}_{17}$ & S18 & 2.2 .2 .5 \\
\hline
\end{tabular}

Procedures for aminomethylation as well as the subsequent $N$-methylation slightly varied with the reactants. In the cases of reactants bearing only a single hydrogen ortho to the hydroxyl group such as bis-(2hydroxyphenyl)methane derivatives $\mathbf{S}_{7}$, a considerable excess of aminomethylation reagent could be used, whereas in all other cases rather stoichiometric amounts and monitoring of reaction progress is necessary to avoid double substitution. Fractions of substance not transformed during the aminomethylation step may in many cases be recovered after $N$-methylation: the tetraalkylammonium salts tend to precipitate leaving the unreacted phenol in solution which may be subjected to the Mannich protocol again. In case of the symmetric bis-(2-hydroxy-5-chloro-phenyl)-methane, products of single and double aminomethylation and unreacted reactant have to be separated by chromatography. ${ }^{8}$ An interesting case is the Mannich reaction of asymmetrically substituted bis-(2-hydroxyphenyl)-methane derivative $\mathbf{S} \mathbf{1 4}$ in which two substitution products are conceivable (see Figure S 2). 


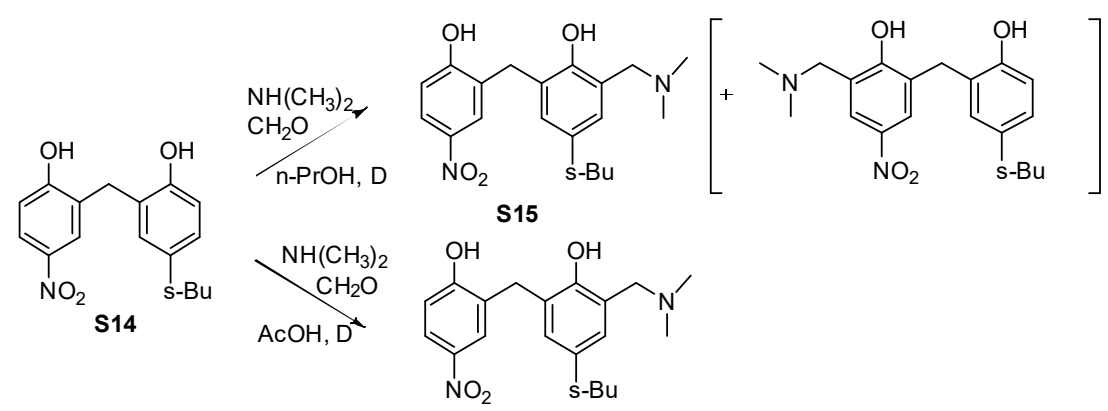

Figure S 2. Considerations concerning regioselectivity in the Mannich reaction of bis-(2-hydroxyphenyl)methane derivative S14.

On the one hand, one may expect preferential aminomethylation of the more electron rich butyl substituted ring. On the other hand, this regioselectivity could be compromised - or even inverted - by a higher tendency of the nitrophenol moiety to be deprotonated by the amine component and thus be converted to a more nucleophilic phenolate motif. Therefore, initial experiments were conducted in which the Mannich reaction was performed in alcoholic solution in the presence of one equivalent of $\mathrm{KOH}$ per hydroxyl group and, alternatively, in glacial acetic acid solution. Indeed, while in the first case a slow conversion to multiple products was observed, under acidic conditions substitution was found to take place rather rapidly and exclusively on the butyl substituted ring.

$\mathrm{N}$-methylation of the Mannich bases was found to be a slow reaction in many cases. Reactivity of the amino groups is probably reduced due to intramolecular OHN hydrogen bonding. This effect can be expected to be strong in Mannich bases with relatively high $\mathrm{OH}$ acidity, and even more so in bis-(2-hydroxyphenyl)-methane derivatives where hydrogen bond cooperativity seems likely:

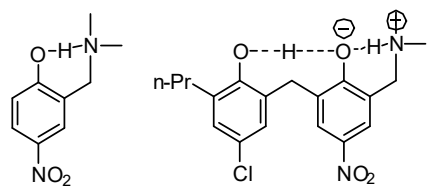

It was found that addition of $\mathrm{KOH}$ or $\mathrm{NaOH}$ was accelerating $N$ alkylation, and in cases such as the generation of $\mathbf{S 8}$ presence of a stoichiometric amount of base turned out to be necessary to obtain quantitative alkylation at room temperature within reasonable time. Competition from $O$ alkylation was not observed under any of the conditions employed. 


\subsubsection{Method c: quaternary ammonium salts to bisphenols}

The key intermediate in $\mathrm{C}-\mathrm{C}$ bond formations to the benzylic carbon atoms are believed to be orthoquinone methides (Figure $\mathrm{S}_{3}$ ) which can be generated in various ways. ${ }^{9}$ Here they were formed either thermally by loss of trimethylamine from the quaternary ammonium salts under basic conditions or by dehydration of salicylic alcohols under acidic conditions. Quinone methides can be intercepted by diverse nucleophiles such as phenols, $\mathrm{H}_{2} \mathrm{O} / \mathrm{OH}^{-}$or cyanide (see section 1.2.4).

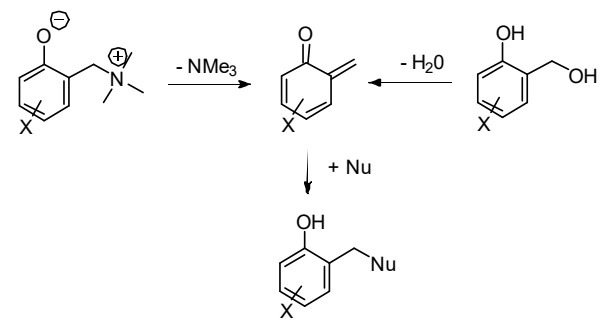

Figure $\mathrm{S}_{3}$. Generation and nucleophilic interception of ortho-quinone methides.

The reaction with water and alcohols is quite slow and thus reactions of quaternary ammonium salts with stronger nucleophiles such as electron rich phenols may be done in aqueous or alcoholic solution.

The bis-(2-hydroxyphenyl)-methane derivative $\mathbf{S 1 4}$ was obtained by coupling of 4-s-butylphenol S13 with tetraalkylammonium iodide according to the scheme in Figure $\mathrm{S} 4$ (procedure in .Section 2.2.3.1).<smiles>CN(C)Cc1cc([N+](=O)[O-])ccc1O</smiles>

S2<smiles>O=C(Sc1ccc(O)cc1)c1ccccc1</smiles>

S13

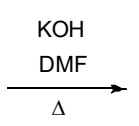

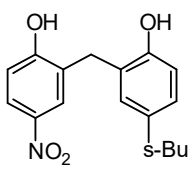

S14

Figure S 4. Coupling of phenols and tetraalkylammonium iodides to bis-(2-hydroxyphenyl)-methanes.

The bis-(2-hydroxyphenyl)-methane derivative $\mathbf{S}_{7}$ was obtained by heating the salicylic alcohol $\mathbf{S}_{\mathbf{4}}$ and phenol S6 in chlorobenzene in the presence of catalytic amounts of a very strong acid (experimental procedure is given in section $2 \cdot 2 \cdot 3 \cdot 3$ ).<smiles>O=[N+]([O-])c1ccc(O)c(CO)c1</smiles>

S4<smiles>CCNc1cc(Cl)ccc1O</smiles>

s6

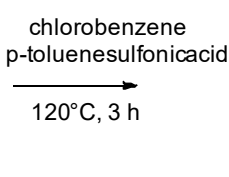<smiles>CCCc1cc(Cl)cc(Cc2cc([N+](=O)[O-])ccc2O)c1O</smiles>

S7

The salicylalcohol 2-hydroxy-5-nitro-phenylmethanol $\mathbf{S}_{\mathbf{4}}$ was obtained by hydrolysis of (2-hydroxy-5nitrobenzyl)-trimethylammonium iodide $\mathbf{S 2}$ (see $\mathbf{2 . 2 . 3 . 2}$ ), but is also commercially available: 


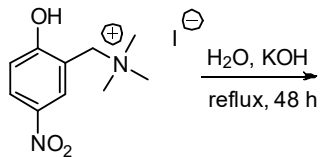

s2<smiles>O=[N+]([O-])c1ccc(O)c(CO)c1</smiles>

S4

\subsubsection{Method d: quaternary ammonium salts to (2-hydroxyphenyl)-acetic acids}

Title compounds listed in Table $\mathrm{S}_{2}$ were obtained according to the scheme in Figure $\mathrm{S}_{5}$. The synthetic route was adapted from Ref. 1 , the use of an aprotic solvent in connection with 18 -crown- 6 as a catalyst in the substitution step was inspired by Ref. 10. Specific procedures can be found in Section $\mathbf{2 . 2}$.
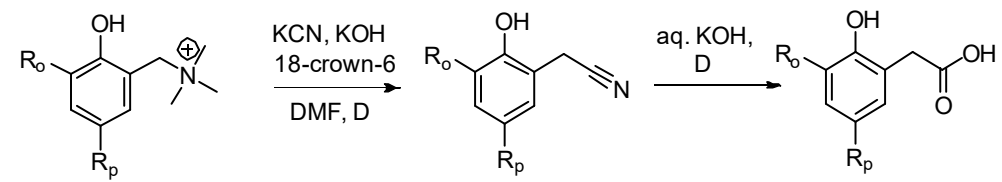

Figure S 5 . Conversion of (2-hydroxyphenyl)-trimethylammonium iodides to (2-hydroxyphenyl)-acetic acids via nitriles. Substituents are given in Table S 2. 
Table $S$ 2. Definitions of substituents $R_{p}$ and $R_{o}$ and nomenclature of the compounds in Figure S6.

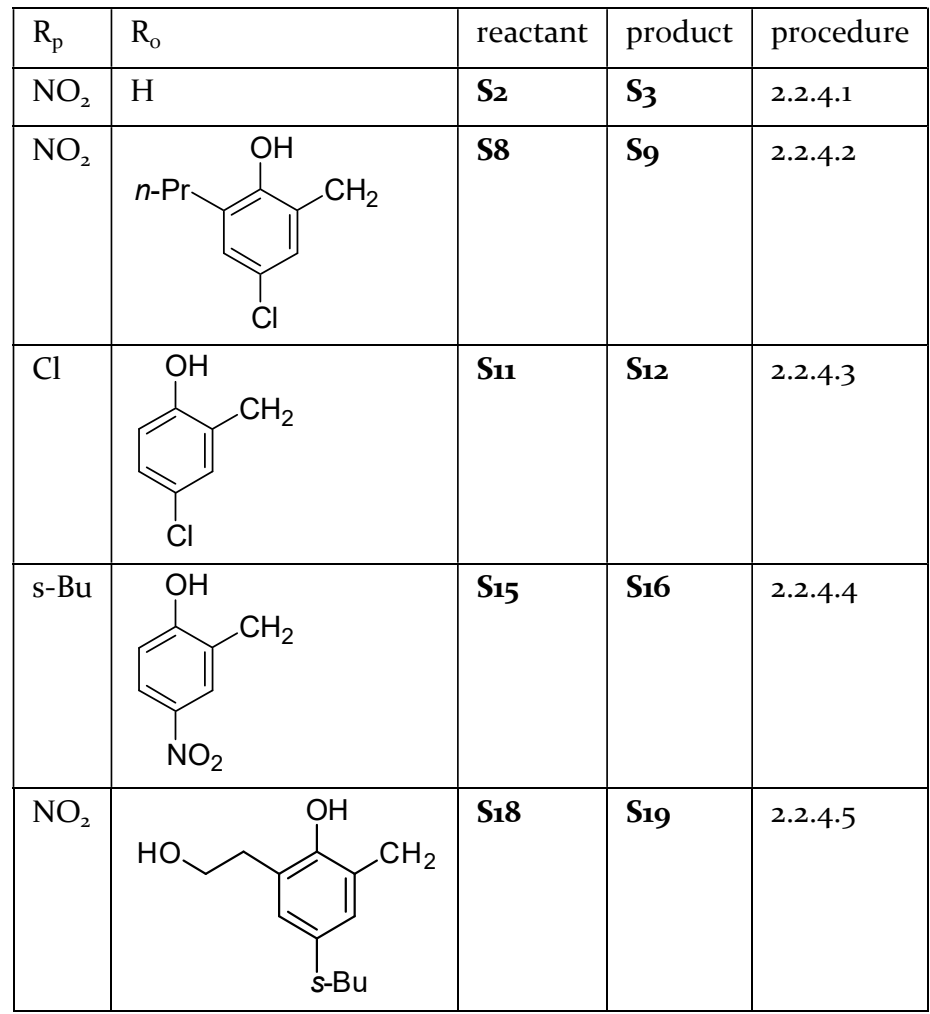

\subsubsection{Method e: reduction to 2-(2-hydroxyphenyl)-ethanol derivatives}

(2-hydroxyphenyl)-acetic acids can be converted to 2-(2-hydroxyphenyl)-ethanols by selective reduction of the carboxylic group with borane. ${ }^{11}$ The reagent is employed conveniently as the dimethylsulfide complex $\mathrm{H}_{3} \mathrm{~B}-\mathrm{S}\left(\mathrm{CH}_{3}\right)_{2}$ in THF solution (procedure in Section 2.2.5.1):

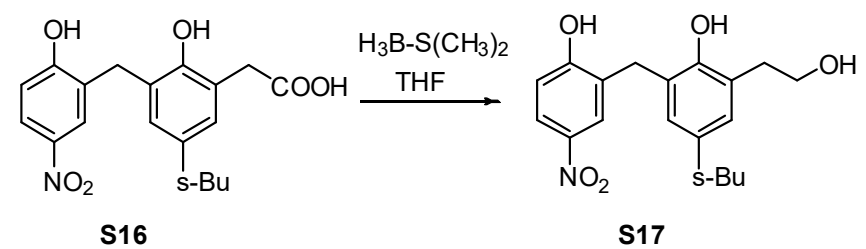




\subsection{Discussion}

It can be summarized that bis-(2-hydroxyphenyl)-methane and (2-hydroxyphenyl)-acetic acid derivatives synthesized according to the given routes are assembled from the corresponding single ring phenolic moieties. Carbon atoms of methylene groups in derivatives of bis-(2-hydroxyphenyl)-methane, (2hydroxyphenyl)-acetic acid and 2-(2-hydroxyphenyl)-ethanol stem from formalin and C-1 atoms of the latter two are introduced as cyanide. In respect to the synthesis of ${ }^{13} \mathrm{C}$ enriched species, this means that labeling within any aromatic ring requires the corresponding phenol. Labeling in those carbon atoms introduced as formalin or cyanide is readily done in the course of the steps discussed here using those common precursors for isotope enrichment.

Yields of bis-(2-hydroxyphenyl)-methanes depended on the substitution pattern of the phenolic nucleophile: in the presence of electron withdrawing groups and a substituent in one of the ortho positions strongly decreased yields were observed. Even with considerable excess of the nucleophile in many cases yields did not exceed $10-20 \%$ in respect to the quinone methide precursor; best yield was $60 \%$ with 4 -sbutylphenol similar to yields of 6o-90\% with 4-methylphenol reported in Ref. 1. In the former cases, isolation of the product from the large variety of side products was not always straightforward. Bis-(2-hydroxyphenyl)methane derivative $\mathbf{S}_{\mathbf{7}}$ was first obtained through the salicyl alcohol (in $17 \%$ yield), as described (see section 2.1.3), after a previous attempts of syntheses from the ammonium salt $\mathbf{S}_{\mathbf{2}}$ had failed. However, $\mathbf{S}_{\mathbf{7}}$ was also obtained analog to $\mathbf{S} \mathbf{1 0}$ (from $\mathbf{S} \mathbf{2}$ in aqueous solution) in approx. 20\% yield. The two alternative coupling protocols with either ammonium salts or salicyl alcohols as quinone methide precursors seem to give similar yields as far as the coupling step itself is concerned. However, the inefficient conversion of the ammonium salts to the salicyl alcohols makes the latter protocol very unattractive. Nonetheless, salicyl alcohols could be key intermediates in alternative syntheses of bis-(2-hydroxyphenyl)-methane derivatives not involving Mannich bases. Salicyl alcohols could be obtained directly from phenols ${ }^{12}$ or by formylation ${ }^{13}$ and subsequent reduction ${ }^{14}$ of the intermediate salicyl aldehyde. 


\section{Experimental Section}

\subsection{DFT calculations}

We used the $\mathrm{B}_{3} \mathrm{LYP} / 6-311++\mathrm{G}^{* *}$ basis set for structure optimization as was employed for similar systems in Ref. ${ }^{15}$. Structures of $\mathbf{1}$ and $\mathbf{2}$ were optimized first. An initial guess for the structure of $\mathbf{3}$ was deduced from these results, however, hydrogen bonding protons were placed in covalent bond distance from phenolic oxygen atoms.

\subsection{Synthetic procedures}

\subsubsection{Chlorophenols}

\subsubsection{2-n-propyl-4-chlorophenol S6}

15.0 g 2-propylphenol ( $\mathbf{S}_{5}, 136.2 \mathrm{~g} / \mathrm{mol}$; $\left.0.11 \mathrm{~mol}\right)$ was dissolved in $50 \mathrm{~mL}$ acetonitrile and $18.3 \mathrm{~g}$ $\mathrm{N}$-chlorosuccinimide $(133.53 \mathrm{~g} / \mathrm{mol} ; 0.14 \mathrm{~mol})$ was added. The solution was stirred overnight at $50^{\circ} \mathrm{C}$. After the quantitative reaction ${ }^{16}$ of 2-propylphenol had been verified by ${ }^{1} \mathrm{H} N \mathrm{NR}$, the solution was decanted from precipitated succinimide and the solvent evaporated. The product $\left(R_{\mathrm{f}}=0.6\right)$ was separated from ortho chlorination products $\left(R_{\mathrm{f}}=0.7\right)$ and other byproducts by column chromatography (silica gel; dichloromethane). 14.1 g of pure $\mathbf{S 6}$ (170.6 g/mol; 75\%) was obtained. ${ }^{1} \mathrm{H}$ NMR (40o MHz, acetone-d6): 6.82 (d, $J=9 \mathrm{~Hz}, 1 \mathrm{H}), 7.00\left(\mathrm{dd}, J_{1}=3 \mathrm{~Hz}, J_{2}=9 \mathrm{~Hz}, 1 \mathrm{H}\right), 7.08(\mathrm{~d}, J=3 \mathrm{~Hz}, 1 \mathrm{H}), 8.47(\mathrm{~s})$.

\subsection{2 (2-hydroxybenzyl)-trimethylammonium iodides}

\subsubsection{1 (2-hydroxy-5-nitro-benzyl)-trimethylammonium iodide $\boldsymbol{S}_{\mathbf{2}}$.}

8.o g 4-nitrophenol (S1, $139 \mathrm{~g} / \mathrm{mol}, 57.6 \mathrm{mmol})$ was dissolved in $20 \mathrm{~mL}$ 1-propanol. $7.3 \mathrm{~mL}$ of a $40 \%$ aqueous solution of dimethylamine $(45.0 \mathrm{~g} / \mathrm{mol}, 7.9 \mathrm{M}, 92 \mathrm{mmol})$ and $4.3 \mathrm{~mL}$ formalin $38 \%(1.1 \mathrm{~g} / \mathrm{mol}, 30.03 \mathrm{~g} / \mathrm{mol}$, 60 mmol ) were added. The mixture was refluxed for 14 hours and then left to cool to r. t. over night. Precipitated product was filtered off and dried in vacuum. 8.o g (71\%) of the Mannich base was obtained. ${ }^{1} \mathrm{H}$ NMR (270 MHz, acetone-d6): $8.07\left(\mathrm{dd}, J_{1}=9 \mathrm{~Hz}, J_{2}=3 \mathrm{~Hz}, 1 \mathrm{H}, \mathrm{H}-4\right), 7.98$ (d, J=3 Hz, $\left.1 \mathrm{H}, \mathrm{H}-6\right), 6.84$ (d, $J=9 \mathrm{~Hz}, 1 \mathrm{H}, \mathrm{H}-3), 3.87\left(\mathrm{~s}, 2 \mathrm{H}, \mathrm{ArC}_{2} \mathrm{~N}\right), 2.40\left(\mathrm{~s}, 6 \mathrm{H}, \mathrm{N}\left(\mathrm{CH}_{3}\right)_{2}\right)$. 
$7.45 \mathrm{~g}$ (38 mmol) 2-((dimethylamino)-methyl)-4-nitrophenol was dissolved in $20 \mathrm{~mL}$ dry THF. $4.75 \mathrm{~mL}$ iodomethane $(2.28 \mathrm{~g} / \mathrm{mL}, 141.9 \mathrm{~g} / \mathrm{mol}, 76 \mathrm{mmol})$ was added and the mixture was stirred for three days at $\mathrm{r}$. $\mathrm{t}$. Solvent and excess iodomethane were removed under reduced pressure. 11.0 g (32.5 mmol, 85\%) of (2Hydroxy-5-nitrophenyl)-trimethylammonium iodide S2 was obtained. ${ }^{1}$ H NMR (270 MHz, DMSO-d6): 8.39 (d, $J=3 \mathrm{~Hz}, 1 \mathrm{H}, \mathrm{Ar}), 8.26\left(\mathrm{dd}, J_{1}=9 \mathrm{~Hz}, J_{2}=3 \mathrm{~Hz}, 1 \mathrm{H}, \mathrm{Ar}\right), 7.12(\mathrm{~d}, J=9 \mathrm{~Hz}, 1 \mathrm{H}, \mathrm{Ar}), 4.54\left(\mathrm{~s}, 2 \mathrm{H}, \operatorname{ArC}_{2} \mathrm{~N}\right), 3.07(\mathrm{~s}$, $\left.9 \mathrm{H}, \mathrm{N}\left(\mathrm{C}_{3}\right)_{3}\right)$.

\subsubsection{Tetraalkylammonium iodide $\mathbf{S 1 1}$}

3.10 g di(2-hydroxy-5-chlorobenzyl)methane $(269.13 \mathrm{~g} / \mathrm{mol} ; 11.5 \mathrm{mmol})$ was heated for 24 hours with $1.46 \mathrm{~mL}$ $40 \%$ aqueous dimethylamine solution (1 eq.) and $0.83 \mathrm{~mL}$ Formalin $38 \%$ (1 eq.) in $25 \mathrm{~mL}$ of n-propanol. After removal of the solvent under reduced pressure, the mixture was suspended in water. The suspension was made acidic $\left(\mathrm{pH}_{2}\right)$ and extracted with dichloromethane to remove unreacted starting material. The aqueous phase was brought to neutral $\mathrm{pH}$ and extracted with ethyl acetate. The organic phase was dried and the solvent was evaporated, resulting in $2.6 \mathrm{~g}$ of a mixture of single $\left(R_{\mathrm{f}}=0.6\right.$ in TLC on silica gel with $\mathrm{CH}_{2} \mathrm{Cl}_{2} /$ methanol 10:1) and double $\left(R_{\mathrm{f}}=0.5\right)$ substitution products and traces of residual starting material $\left(R_{\mathrm{f}}=0.8\right)$. These were separated by column chromatography (same phases as in TLC), yielding 1.60 g product of single aminomethylation $(326 \mathrm{~g} / \mathrm{mol}, 4.9 \mathrm{mmol}, 39 \%)$ and $0.53 \mathrm{~g}$ by-product di(2-hydroxy-3-(2(dimethylamino)-ethyl)-5-chlorobenzyl)methane.

Mannich base (product of single aminomethylation): ${ }^{1} \mathrm{H}$ NMR (270 MHz, methanol-d4): 7.01-6.99 (convoluted, $2 \mathrm{H}, \underline{\left.\operatorname{ArCH}_{2} \mathrm{~N}\right),} 6.98\left(\mathrm{dd}, J_{1}=9 \mathrm{~Hz}, J_{2}=3 \mathrm{~Hz}, 1 \mathrm{H}, \quad \mathrm{CHC} \underline{\mathrm{HCClCH}}\right), 6.88(\mathrm{~d}, J=3 \mathrm{~Hz}, 1 \mathrm{H}$, $\mathrm{CHCHCClC} \underline{\mathrm{H}}), 6.72\left(\mathrm{~d}, J=9 \mathrm{~Hz},{ }_{1} \mathrm{H}, \mathrm{C} \underline{\mathrm{HCHCClCH}}\right), 3.80\left(\mathrm{~s}, 2 \mathrm{H}, \operatorname{ArC}_{2} \underline{\mathrm{Hr}}_{2}\right), 3.70\left(\mathrm{~s}, 2 \mathrm{H}, \operatorname{ArC}_{2} \mathrm{~N}\right), 2.39(\mathrm{~s}, 6 \mathrm{H}$, $\left.\left(\mathrm{CH}_{3}\right)_{2}\right)$. Di(2-hydroxy-3-(2-(dimetylamino)ethyl)-5-chlorobenzyl)methane: ${ }^{1} \mathrm{H}$ NMR (27o MHz, methanol-d4): $6.99(\mathrm{~d}, J=3 \mathrm{~Hz}, 2 \mathrm{H}, \mathrm{Ar}), 6.93(\mathrm{~d}, J=3 \mathrm{~Hz}, 2 \mathrm{H}, \mathrm{Ar}), 3.87\left(\mathrm{~s}, 2 \mathrm{H}, \operatorname{ArCH}_{2} \mathrm{Ar}\right), 3.70(\mathrm{~s}, 4 \mathrm{H}, \operatorname{ArCH} \mathrm{N}), 2.39(\mathrm{~s}, 12 \mathrm{H}$, $\left.\left(\mathrm{CH}_{3}\right)_{2}\right)$.

The Mannich base was dissolved in THF and stirred with $0.90 \mathrm{~mL}$ of iodomethane (3 eq.) until TLC indicated complete conversion (one week). Solvent and excess reagent were evaporated under reduced pressure, leaving the sufficiently pure iodide in almost quantitative yield. ${ }^{1} \mathrm{H}$ NMR (270 MHz, DMSO-d6): 7·37 $\left(\mathrm{d}, J=3 \mathrm{~Hz}, 1 \mathrm{H}, \mathrm{CHCClCHCCH}_{2}\right), 7 \cdot 11\left(\mathrm{~d}, J=3 \mathrm{~Hz}, 1 \mathrm{H}, \mathrm{CHCClCHCCH}_{2}\right), 7.11\left(\mathrm{dd}, J_{1}=9 \mathrm{~Hz}, J_{2}=3 \mathrm{~Hz}, 1 \mathrm{H}\right.$, $\mathrm{CHC} \underline{\mathrm{HCClCH}}), 7.03(\mathrm{~d}, J=3 \mathrm{~Hz}, 1 \mathrm{H}, \mathrm{CHCHCClC} \underline{\mathrm{H}}), 6.84(\mathrm{~d}, J=9 \mathrm{~Hz}, 1 \mathrm{H}, \underline{\mathrm{CHCHCClCH}}), 4.50\left(\mathrm{~s}, 2 \mathrm{H}, \mathrm{ArCH}_{2} \mathrm{~N}\right)$, $3.89\left(\mathrm{~s}, 2 \mathrm{H}, \mathrm{ArCH}_{2} \mathrm{Ar}\right), 3.05\left(\mathrm{~s}, 6 \mathrm{H},\left(\mathrm{CH}_{3}\right)_{3}\right)$. 


\subsubsection{Tetraalkylammonium iodide $\mathbf{S 8}$}

$1.50 \mathrm{~g}$ of $\mathrm{S}_{7}(321.8 \mathrm{~g} / \mathrm{mol}, 4.66 \mathrm{mmol})$ was treated for 12 hours with 1.2 equivalents of each $37 \%$ formalin and $40 \%$ aqueous dimethylamine solution in $10 \mathrm{~mL} \mathrm{n}$-propanol. The Mannich base precipitated from the alcoholic solution; $1.47 \mathrm{~g}$ was collected by filtration after the reaction mixture had been stirred over night at r. t. ${ }^{1} \mathrm{H}$ NMR $\quad\left(250 \mathrm{MHz}, \quad\right.$ acetone-d6): $8.19 \quad\left(\mathrm{~d}, J=3 \mathrm{~Hz}, \quad{ }_{1} \mathrm{H}, \quad \mathrm{CH} 2 \mathrm{CCHC}\left(\mathrm{NO}_{2}\right) \mathrm{C} \underline{\mathrm{H}}\right), \quad 7.87 \quad(\mathrm{~d}, \quad J=3 \mathrm{~Hz}, \quad 1 \mathrm{H}$, $\left.\mathrm{CH}_{2} \mathrm{CCH} ! \mathrm{C}\left(\mathrm{NO}_{2}\right) \mathrm{CH}\right), 7.20\left(\mathrm{~d}, J=3 \mathrm{~Hz}, 1 \mathrm{H}, \mathrm{CH}_{2} \mathrm{CCHCClC} \underline{\mathrm{H}}\right), 6.87\left(\mathrm{~d}, J=3 \mathrm{~Hz}, 1 \mathrm{H}, \mathrm{CH}_{2} \mathrm{CC} \underline{\mathrm{HCClCH}}\right), 4.26(\mathrm{~s}$, $\left.2 \mathrm{H}, \operatorname{ArC} \underline{H}_{2} \mathrm{Ar}\right), 3.8 \mathrm{o}\left(\mathrm{s}, 2 \mathrm{H}, \mathrm{ArCH}_{2} \mathrm{~N}\right), 2.95\left(\mathrm{~s}, 6 \mathrm{H}, \mathrm{N}\left(\mathrm{CH}_{3}\right)_{2}\right), 2.52\left(\mathrm{t}, J=7 \mathrm{~Hz}, 2 \mathrm{H}, \mathrm{CH}_{2} \mathrm{CH}_{2} \mathrm{CH}_{3}\right), 1.55(\mathrm{qt}$, $\left.J_{1}=7 \mathrm{~Hz}, J_{2}=7 \mathrm{~Hz}, 2 \mathrm{H}, \mathrm{CH}_{2} \underline{\mathrm{H}}_{2} \mathrm{CH}_{3}\right), 0.89\left(\mathrm{t}, J=7 \mathrm{~Hz}, 3 \mathrm{H}, \mathrm{CH}_{2} \mathrm{CH}_{2} \underline{\mathrm{CH}}_{3}\right)$.

The Mannich base (o.66 g, 378.9 g/mol, $1.74 \mathrm{mmol}$ ) and $0.11 \mathrm{~g}$ of $\mathrm{KOH}$ (1.2 eq.) were suspended in $10 \mathrm{~mL}$ acetonitrile and $1.0 \mathrm{~mL}$ iodomethane (ca. 10 eq.). This mixture was stirred at r. t. until TLC (silica gel, ethyl acetate $+5 \%$ formic acid) indicated almost complete conversion of the Mannich base (two days). The solvent and excess reagent were removed under reduced pressure, and the residue was washed with ethyl acetate. $0.87 \mathrm{~g}$ of a yellow substance quite soluble in acetone was obtained. ${ }^{17} \mathrm{H}$ NMR (250 MHz, acetone-d6): 8.16 (d, $\left.J=3 \mathrm{~Hz}, \quad{ }_{1 \mathrm{H}}, \mathrm{CH}_{2} \mathrm{CCHC}\left(\mathrm{NO}_{2}\right) \mathrm{C} \underline{\mathrm{H}}\right), \quad 8.09\left(\mathrm{~d}, J=3 \mathrm{~Hz}, \quad 1 \mathrm{H}, \quad \mathrm{CH}_{2} \mathrm{CC} \underline{\mathrm{HC}}\left(\mathrm{NO}_{2}\right) \mathrm{CH}\right), \quad 7.18 \quad(\mathrm{~d}, \quad J=3 \mathrm{~Hz}, \quad 1 \mathrm{H}$, $\left.\mathrm{CH}_{2} \mathrm{CCHCClC} \underline{\mathrm{H}}\right), 6.84\left(\mathrm{~d}, J=3 \mathrm{~Hz},{ }_{1} \mathrm{H}, \mathrm{CH}_{2} \mathrm{CC} \underline{\mathrm{HCClCH}}\right), 4.51\left(\mathrm{~s}, 2 \mathrm{H}, \mathrm{ArC}_{2} \mathrm{~N}\right), 3.80\left(\mathrm{~s}, 2 \mathrm{H}, \operatorname{ArC}_{2} \mathrm{Ar}\right), 3.25(\mathrm{~s}$, $\left.9 \mathrm{H}, \mathrm{N}\left(\mathrm{CH}_{3}\right)_{3}\right), 2.51\left(\mathrm{t}, J=7 \mathrm{~Hz}, 2 \mathrm{H}, \underline{\mathrm{C}}_{2} \mathrm{CH}_{2} \mathrm{CH}_{3}\right), 1.54\left(\mathrm{qt}, J_{1}=7 \mathrm{~Hz}, J_{2}=7 \mathrm{~Hz}, 2 \mathrm{H}, \mathrm{CH}_{2} \mathrm{C}_{2} \mathrm{CH}_{3}\right), 0.88(\mathrm{t}, J=7 \mathrm{~Hz}$, $\left.3 \mathrm{H}, \mathrm{CH}_{2} \mathrm{CH}_{2} \underline{\mathrm{CH}}_{3}\right)$.

\subsubsection{Tetraalkylammonium iodide $\mathbf{S 1 5}$}

$4.30 \mathrm{~mL}$ formalin $38 \%(13.9 \mathrm{M}, 59.6 \mathrm{mmol})$ and $8.2 \mathrm{~mL} 40 \%$ aqueous solution of dimethylamine (7.9 M, $65 \mathrm{mmol}$ ) were mixed under stirring. A rapid exothermic reaction was observed. After the mixture had cooled to ambient temperature (one hour), it was added to a solution of $15.0 \mathrm{~g}$ of bis-(2-hydroxyphenyl)-methane $\mathbf{S} \mathbf{4}$ ( $301.34 \mathrm{~g} / \mathrm{mol}, 49.8 \mathrm{mmol})$ in $150 \mathrm{~mL}$ glacial acetic acid. This mixture was stirred and heated to $100^{\circ} \mathrm{C}$ for 10 hours. Solvent, water and possible excess reagent were removed under reduced pressure, leaving a light yellow oil. By column chromatography (silica gel, ethyl acetate/methanol 2:1) the desired Mannich base $\left(R_{\mathrm{f}}=0.3\right)$ was separated from starting material $\mathbf{S 1 4}\left(R_{\mathrm{f}}=0.9\right)$ and side products $\left(R_{\mathrm{f}}<<0.3\right) .13 .84 \mathrm{~g}$ (358.4 g/mol, $38.62 \mathrm{mmol}, 78 \%)$ of the precursor of S15 were obtained. ${ }^{1} \mathrm{H}$ NMR (50o MHz, acetone-d6): 8.12 $\left(\mathrm{d}, J=3 \mathrm{~Hz}, 1 \mathrm{H}, \mathrm{CHCHC}\left(\mathrm{NO}_{2}\right) \mathrm{C} \underline{\mathrm{H}}\right), 7.90\left(\mathrm{dd}, J_{1}=9 \mathrm{~Hz}, J_{2}=3 \mathrm{~Hz}, \mathrm{CHC} \underline{\mathrm{HC}}\left(\mathrm{NO}_{2}\right) \mathrm{CH}\right), 7.25(\mathrm{~d}, J=3 \mathrm{~Hz}, 1 \mathrm{H}$, $\mathrm{CHC}(\mathrm{s}-\mathrm{Bu}) \mathrm{CH}), 6.92(\mathrm{~d}, J=3 \mathrm{~Hz}, 1 \mathrm{H}, \mathrm{CHC}(\mathrm{s}-\mathrm{Bu}) \mathrm{CH}), 6.71\left(\mathrm{~d}, J=9 \mathrm{~Hz}, \mathrm{C} \underline{\mathrm{HCHC}}\left(\mathrm{NO}_{2}\right) \mathrm{CH}\right), 4.18(\mathrm{~s}, 2 \mathrm{H}$, 
$\left.\operatorname{ArC} \underline{H}_{2} \mathrm{Ar}\right), 3.90\left(\mathrm{~s}, 2 \mathrm{H}, \operatorname{ArCH}_{2} \mathrm{~N}\right), 2.79\left(\mathrm{~s}, 6 \mathrm{H}, \mathrm{N}\left(\mathrm{C}_{3}\right)_{2}\right), 2.48\left(\mathrm{tq}, J_{1}=J_{2}=7 \mathrm{~Hz}, 1 \mathrm{H}, \mathrm{CH}_{3} \underline{\mathrm{HCH}}_{2}\right), 1.51(\mathrm{qd}$, $\left.J_{1}=J_{2}=7 \mathrm{~Hz}, 2 \mathrm{H}, \mathrm{CHC}_{2} \mathrm{CH}_{3}\right), 1.15\left(\mathrm{~d}, J=7 \mathrm{~Hz},{ }_{3} \mathrm{H}, \mathrm{CHC} \underline{H}_{3}\right), 0.76\left(\mathrm{t}, J=8 \mathrm{~Hz}, 3 \mathrm{H}, \mathrm{CH}_{2} \underline{\mathrm{C}}_{3}\right)$.

$13.8 \mathrm{~g}$ of the precursor of $\mathbf{S} \mathbf{1 5}(38.5 \mathrm{mmol})$ was dissolved in $50 \mathrm{~mL}$ of THF. $5 \mathrm{~mL}$ of iodomethane (>2 eq.) were added and the mixture was stirred at r. t. until TLC indicated complete conversion of the Mannich base (one week). Solvent and excess reagent were removed under reduced pressure, leaving $17.7 \mathrm{~g}(92 \%)$ of the tetraalkylammonium salt $\mathbf{S 1 5}$ crystallizing as a foam. ${ }^{1} \mathrm{H}$ NMR (270 MHz, DMSO-d6): 7.99 (dd, $J_{1}=9 \mathrm{~Hz}$, $\left.J_{2}=3 \mathrm{~Hz}, \mathrm{CHC} \underline{\mathrm{HC}}\left(\mathrm{NO}_{2}\right) \mathrm{CH}\right), 7.73\left(\mathrm{~d}, J=3 \mathrm{~Hz}, 1 \mathrm{H}, \mathrm{CHCHC}\left(\mathrm{NO}_{2}\right) \mathrm{C} \underline{\mathrm{H}}\right), 7.15(\mathrm{~d}, J=3 \mathrm{~Hz}, 1 \mathrm{H}, \mathrm{CHC}(\mathrm{s}-\mathrm{Bu}) \mathrm{CH}), 7.10$ $(\mathrm{d}, J=3 \mathrm{~Hz}, 1 \mathrm{H}, \mathrm{CHC}(\mathrm{s}-\mathrm{Bu}) \mathrm{CH}), 6.95\left(\mathrm{~d}, J=9 \mathrm{~Hz}, \mathrm{C} \underline{\mathrm{HCHC}}\left(\mathrm{NO}_{2}\right) \mathrm{CH}\right), 4.53\left(\mathrm{~s}, 2 \mathrm{H}, \operatorname{ArC}_{2} \mathrm{~N}\right), 3.96(\mathrm{~s}, 2 \mathrm{H}$, $\left.\operatorname{ArC} \underline{H}_{2} \mathrm{Ar}\right), \quad 3.04\left(\mathrm{~s}, 9 \mathrm{H}, \quad \mathrm{N}\left(\mathrm{CH}_{3}\right)_{3}\right), 2.51\left(\mathrm{tq}, J_{1}=J_{2}=7 \mathrm{~Hz}, 1 \mathrm{H}, \quad \mathrm{CH}_{3} \mathrm{CHCH}_{2}\right), 1.49 \quad\left(\mathrm{qd}, J_{1}=J_{2}=7 \mathrm{~Hz}, 2 \mathrm{H}\right.$, $\left.\mathrm{CHC}_{2} \mathrm{CH}_{3}\right), 1.14\left(\mathrm{~d}, J=7 \mathrm{~Hz},{ }_{3} \mathrm{H}, \mathrm{CHC}_{3}\right), 0.74\left(\mathrm{t}, J=8 \mathrm{~Hz},{ }_{3} \mathrm{H}, \mathrm{CH}_{2} \underline{\mathrm{C}}_{3}\right)$.

\subsubsection{Tetraalkylammonium iodide $\mathbf{S} \mathbf{8}$}

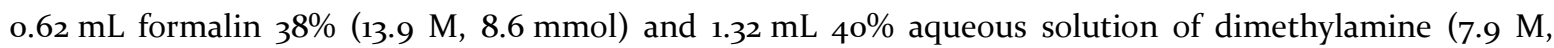
$10.4 \mathrm{mmol}$ ) were mixed. A rapid exothermic reaction was observed. After the mixture had cooled to ambient temperature, it was added to a suspension of $2.0 \mathrm{~g}$ of bis-(2-hydroxyphenyl)-methane S17 (345.4 g/mol, $5.79 \mathrm{mmol}$ ) in n-propanol. This mixture was stirred and heated to $90^{\circ} \mathrm{C}$. By $\mathrm{TLC}$ (silica gel, dichloromethane/methanol 10:1), aminoalkylation of $\mathbf{S} 17\left(R_{\mathrm{f}}=0.75\right)$ to the Mannich base $\left(R_{\mathrm{f}}=0.25\right)$ was monitored. After 10 hours, approx. half conversion was detected. A second batch of reagent mixture as described above was added. Another 10 hours later the conversion was complete. Solvent, excess reagent and water were removed under reduced pressure leaving the sufficiently pure Mannich base (2.40 g, 402.5 g/mol, $5.96 \mathrm{mmol}, \sim 100 \%)$ as a foamy yellow residue. ${ }^{1} \mathrm{H}$ NMR $(250 \mathrm{MHz}$, acetone-d6): $8.12(\mathrm{~d}, J=3 \mathrm{~Hz}, 1 \mathrm{H}$, $\left.\mathrm{CHC}\left(\mathrm{NO}_{2}\right) \mathrm{CH}\right), 7.86\left(\mathrm{~d}, J=3 \mathrm{~Hz},{ }_{1} \mathrm{H}, \mathrm{CHC}\left(\mathrm{NO}_{2}\right) \mathrm{CH}\right), 7.01(\mathrm{~s}, 1 \mathrm{H}, \mathrm{CHC}(\mathrm{s}-\mathrm{Bu}) \mathrm{CH}), 6.77(\mathrm{~s}, 1 \mathrm{H}, \mathrm{CHC}(\mathrm{s}-\mathrm{Bu}) \mathrm{CH})$, $4.22\left(\mathrm{~s}, 2 \mathrm{H}, \operatorname{ArC} \underline{H}_{2} \mathrm{Ar}\right), 3.83\left(\mathrm{~s}, 2 \mathrm{H}, \mathrm{ArCH}_{2} \mathrm{~N}\right), 3.72\left(\mathrm{t}, J=6 \mathrm{~Hz}, 2 \mathrm{H}, \mathrm{CH}_{2} \mathrm{CH}_{2} \mathrm{OH}\right), 2.89\left(\mathrm{~s}, 6 \mathrm{H}, \mathrm{N}\left(\mathrm{CH}_{3}\right)_{2}\right), 2.81(\mathrm{t}$, $\left.J=6 \mathrm{~Hz}, 2 \mathrm{H}, \mathrm{CH}_{2} \underline{\mathrm{H}}_{2} \mathrm{OH}\right), 2.45\left(\mathrm{qt}, J_{1}=J_{2}=7 \mathrm{~Hz}, 1 \mathrm{H}, \mathrm{CH}_{3} \underline{\mathrm{CHCH}}_{2}\right), 1.52\left(\mathrm{dq}, J_{1}=J_{2}=7 \mathrm{~Hz}, 2 \mathrm{H}, \mathrm{CHC}_{2} \mathrm{CH}_{3}\right), 1.16$ $\left(\mathrm{d}, J=7 \mathrm{~Hz},{ }_{3} \mathrm{H}, \mathrm{CHC}_{3}\right), 0.78\left(\mathrm{t}, J=8 \mathrm{~Hz},{ }_{3} \mathrm{H}, \mathrm{CH}_{2} \underline{\mathrm{C}}_{3}\right)$.

The Mannich base $(2.40 \mathrm{~g}, 402.5 \mathrm{~g} / \mathrm{mol}, 5.96 \mathrm{mmol})$ was dissolved in THF, and $0.75 .5 \mathrm{~mL}$ iodomethane (2.5 eq.) and $0.34 \mathrm{~g} \mathrm{KOH} \mathrm{(1} \mathrm{eq.)} \mathrm{were} \mathrm{added.} \mathrm{This} \mathrm{mixture} \mathrm{was} \mathrm{stirred} \mathrm{at} \mathrm{r.} \mathrm{t.} \mathrm{for} \mathrm{one} \mathrm{week.} \mathrm{After} \mathrm{evaporation} \mathrm{of}$ solvent and excess reagent an orange colored foam was obtained. This was taken up in acetone. A small amount of an insoluble component (potassium iodide?) was filtered off. After evaporation of the solvent, 4.o g of substance remained. ${ }^{1} \mathrm{H}$ NMR (250 MHz, DMSO-d6): $8.14\left(\mathrm{~d}, J=3 \mathrm{~Hz}, 1 \mathrm{H}, \mathrm{CHC}\left(\mathrm{NO}_{2}\right) \mathrm{CH}\right), 8.00(\mathrm{~d}, J=3 \mathrm{~Hz}$, 
$\left.{ }_{1} \mathrm{H}, \mathrm{CHC}\left(\mathrm{NO}_{2}\right) \mathrm{CH}\right), 6.87(\mathrm{~d}, J=3 \mathrm{~Hz}, 1 \mathrm{H}, \mathrm{CHC}(\mathrm{s}-\mathrm{Bu}) \mathrm{CH}), 6.73(\mathrm{~d}, J=3 \mathrm{~Hz}, 1 \mathrm{H}, \mathrm{CHC}(\mathrm{s}-\mathrm{Bu}) \mathrm{CH}), 4.45(\mathrm{~s}, 2 \mathrm{H}$, $\left.\operatorname{ArC} \underline{H}_{2} \mathrm{~N}\right), 3.79\left(\mathrm{~s}, 2 \mathrm{H}, \operatorname{ArC} \underline{\mathrm{H}}_{2} \mathrm{Ar}\right), 3.52\left(\mathrm{t}, J=6 \mathrm{~Hz}, 2 \mathrm{H}, \underline{\mathrm{C}}_{2} \mathrm{CH}_{2} \mathrm{OH}\right), 3.02\left(\mathrm{~s}, 9 \mathrm{H}, \mathrm{N}\left(\mathrm{C}_{3}\right)_{3}\right), 2.67(\mathrm{t}, J=6 \mathrm{~Hz}, 2 \mathrm{H}$, $\mathrm{CH}_{2} \underline{\mathrm{C}}_{2} \mathrm{OH}$ ), $2.40\left(\mathrm{qt}, J_{1}=J_{2}=7 \mathrm{~Hz}, 1 \mathrm{H}, \mathrm{CH}_{3} \underline{\mathrm{CHCH}}_{2}\right), 1.45\left(\mathrm{dq}, J_{1}=J_{2}=7 \mathrm{~Hz}, 2 \mathrm{H}, \mathrm{CHCH}_{2} \mathrm{CH}_{3}\right), 1.09(\mathrm{~d}, J=7 \mathrm{~Hz}$, $\left.3 \mathrm{H}, \mathrm{CHC}_{3}\right), 0.73\left(\mathrm{t}, J=8 \mathrm{~Hz}, 3 \mathrm{H}, \mathrm{CH}_{2} \underline{\mathrm{CH}}_{3}\right)$.

\subsubsection{Bis-(2-hydroxyphenyl)methanes (bisphenols)}

$$
\text { (2-hydroxy-5-nitrobenzyl)-(2-hydroxy-5-s-butly)-methane ( } \left.\mathbf{S 1 4}_{\mathbf{4}}\right)
$$

40.0 g of (2-hydroxy-5-nitrobenzyl)-trimethylammonium iodide (S2, $338 \mathrm{~g} / \mathrm{mol}, 118 \mathrm{mmol})$ and $35.4 \mathrm{~g} \mathrm{4-s-}$ butylphenol (150.2 g/mol, $236 \mathrm{mmol})$ were suspended in $250 \mathrm{~mL}$ of water. $18.9 \mathrm{~g} \mathrm{NaOH}(40 \mathrm{~g} / \mathrm{mol}, 472 \mathrm{mmol})$ were added and the mixture was refluxed $\left(\mathrm{PEG}\right.$ bath $120^{\circ} \mathrm{C}$ ) for 20 hours. Then the solution was made acidic and steam distilled until no more 4 -s-butylphenol was carried over ( 3 liters of water). Finally, the residue was concentrated to approx. $200 \mathrm{~mL}$ water volume and extracted with ethyl acetate. The organic phase was dried and the solvent evaporated. The dark residue contained almost exclusively the desired product. Recrystallization from toluene readily furnished sufficiently pure $\mathbf{S 1 4}$ (21.1 g, 301.34 g/mol, 70.0 mmol, 6o\%). ${ }^{1} \mathrm{H}$ NMR (250 MHz, acetone-d6): 8.0o $\left(\mathrm{d}, J=3 \mathrm{~Hz}, 1 \mathrm{H}, \mathrm{CHCHC}\left(\mathrm{NO}_{2}\right) \mathrm{C} \underline{\mathrm{H}}\right), 7.98\left(\mathrm{dd}, J_{1}=8 \mathrm{~Hz}, J_{2}=3 \mathrm{~Hz}\right.$, $\left.\mathrm{CHC} \underline{\mathrm{HC}}\left(\mathrm{NO}_{2}\right) \mathrm{CH}\right), 7.08\left(\mathrm{~d}, J=3 \mathrm{~Hz},{ }_{1} \mathrm{H}, \mathrm{CHCHC}(s-\mathrm{Bu}) \mathrm{C} \underline{\mathrm{H}}\right), 7.03\left(\mathrm{~d}, J=8 \mathrm{~Hz}, \mathrm{C} \underline{\mathrm{HCHC}}\left(\mathrm{NO}_{2}\right) \mathrm{CH}\right), 6.94(\mathrm{dd}$, $\left.J_{1}=3 \mathrm{~Hz}, J_{2}=8 \mathrm{~Hz}, 1 \mathrm{H}, \mathrm{CHC} \underline{\mathrm{HC}}(s-\mathrm{Bu}) \mathrm{CH}\right), 6.83(\mathrm{~d}, J=8 \mathrm{~Hz}, 1 \mathrm{H}, \mathrm{CHCHC}(s-\mathrm{Bu}) \mathrm{C} \underline{\mathrm{H}}), 4.01\left(\mathrm{~s}, 2 \mathrm{H}, \operatorname{ArC} \underline{H}_{2} \mathrm{Ar}\right), 2.48$ $\left(\mathrm{qt}, J_{1}=J_{2}=7 \mathrm{~Hz}, 1 \mathrm{H}, \mathrm{CH}_{3} \underline{\mathrm{HCH}}_{2}\right), 1.52\left(\mathrm{dq}, J_{1}=J_{2}=7 \mathrm{~Hz}, 2 \mathrm{H}, \mathrm{CHCH}_{2} \mathrm{CH}_{3}\right), 1.16\left(\mathrm{~d}, J=7 \mathrm{~Hz}, 3 \mathrm{H}, \mathrm{CHC} \underline{\mathrm{H}}_{3}\right), 0.77$ $\left(\mathrm{t}, J=7 \mathrm{~Hz},{ }_{3} \mathrm{H}, \mathrm{CH}_{2} \underline{\mathrm{C}}_{3}\right)$.

\subsubsection{2 (2-hydroxy-5-nitro-phenyl)-methanol ( $\left.\boldsymbol{S}_{4} \boldsymbol{a}\right)$}

In a flask equipped with inert gas inlet and a reflux condenser attached to a bubble counter, $10 \mathrm{~g}$ of (2hydroxy-5-nitrobenzyl)-trimethylammonium iodide $(29.6 \mathrm{mmol})$ were dissolved in solution prepared from $30 \mathrm{~g} \mathrm{KOH}$ and $100 \mathrm{~mL}$ water and heated at reflux. Progress of the reaction was monitored by checking for trimethylamine in the exiting inert gas stream by a wet $\mathrm{pH}$ paper. After gas evolution had ceased ( 2 days), the solution was acidified and the mixture was extracted with ethyl acetate several times. The combined organic phases were dried with sodium sulfate and the solvent was evaporated. 4.0 g raw product were obtained that were subjected to column chromatography (silica gel; dichloromethane/methanol 10:1). $1.6 \mathrm{~g}$ (9.5 mmol; 32\%) (2-hydroxy-5-nitro-phenyl)methanol were obtained. 


\subsubsection{3 (2-hydroxy-5-nitrobenzyl)-(2-hydroxy-3-propyl-5-chlorobenzyl)- methane $\left(S_{7}\right)$}

$4.8 \mathrm{~g}$ 2-hydroxy-5-nitro-phenylmethanol $(28 \mathrm{mmol})$ and $5.3 \mathrm{~g}$ 2-propyl-4-chlorophenol (31 $\mathrm{mmol})$ in $8 \mathrm{~mL}$ chlorobenzene were stirred and heated to $120^{\circ} \mathrm{C}$. After a homogenous mixture had been obtained, a catalytic amount of 4-toluenesulfonic acid (0.05 g, $1.3 \mathrm{mmol}$ ) was added. After three hours, the solvent was distilled off in high vacuum. The residue was dissolved in ethyl acetate. The solution was washed with water and dried. Column chromatography (silica gel; dichloromethane/methanol 20:1) afforded $2.0 \mathrm{~g}$ of a brown product. This brown product was treated with as much refluxing dichloromethane as was necessary to completely dissolve the dark byproducts. The solution was separated from the colorless crystalline product (2-hydroxy-5nitrobenzyl)-(2-hydroxy-3-propyl-5-chlorobenzyl)methane: $1.5 \mathrm{~g} \quad(4.7 \mathrm{mmol}, \quad 17 \%) . \quad{ }^{1} \mathrm{H} \quad \mathrm{NMR} \quad(250 \mathrm{MHz}$, acetone-d6): $8.05\left(\mathrm{~d}, J=3 \mathrm{~Hz},{ }_{1} \mathrm{H}, \mathrm{CHCHC}\left(\mathrm{NO}_{2}\right) \mathrm{CH}\right)$, 8.oo (dd, $\left.J_{1}=9 \mathrm{~Hz}, J_{2}=3 \mathrm{~Hz}, 1 \mathrm{H}, \mathrm{CHC} \underline{H C}\left(\mathrm{NO}_{2}\right) \mathrm{CH}\right)$, 7.06 $(\mathrm{d}, J=3 \mathrm{~Hz}, 1 \mathrm{H}, \mathrm{CHCClCH}), 7.04\left(\mathrm{~d}, J=9 \mathrm{~Hz}, 1 \mathrm{H}, \mathrm{C} \underline{\mathrm{HCHC}}\left(\mathrm{NO}_{2}\right) \mathrm{CH}\right), 7.01(\mathrm{~d}, J=3 \mathrm{~Hz}, 1 \mathrm{H}, \mathrm{CHCClCH}), 4.03(\mathrm{~s}$, $\left.2 \mathrm{H}, \operatorname{ArC} \underline{H}_{2} \mathrm{Ar}\right), 2.62\left(\mathrm{t}, J=7 \mathrm{~Hz}, 2 \mathrm{H}, \underline{\mathrm{C}}_{2} \mathrm{CH}_{2} \mathrm{CH}_{3}\right), 1.60\left(\mathrm{qt}, J_{1}=7 \mathrm{~Hz}, J_{2}=7 \mathrm{~Hz}, 2 \mathrm{H}, \mathrm{CH}_{2} \underline{\mathrm{CH}}_{2} \mathrm{CH}_{3}\right), 0.93(\mathrm{t}$, $\left.J=7 \mathrm{~Hz},{ }_{3} \mathrm{H}, \mathrm{CH}_{2} \mathrm{CH}_{2} \underline{\mathrm{C}}_{3}\right)$.

\subsection{4 (2-hydroxyphenyl)-acetic acids}

\subsubsection{2-(2-hydroxy-5-nitrophenyl)-acetic acid $\left(S_{3}\right)$}

In a flask equipped with inert gas inlet, a reflux condenser attached to a bubble counter, 3.o g (8.9 mmol) (2-hydroxy-5-chloro-benzyl)-trimethylammonium iodide was stirred and heated to $100^{\circ} \mathrm{C}$ with $0.5 \mathrm{~g} \mathrm{KOH}$ (9 mmol) and $0.87 \mathrm{~g}(13 \mathrm{mmol}) \mathrm{KCN}$ in the presence of $0.35 \mathrm{~g} 18$-crown-6 (15\%) in DMF solution (10 $\mathrm{mL}$ ). Progress of the reaction was monitored by checking for trimethylamine in the exiting inert gas stream by a wet $\mathrm{pH}$ paper. After evolution of trimethylamine had ceased ( 5 hours), DMF was distilled off in high vacuum. The residue was taken up in water, the $\mathrm{pH}$ was adjusted to slightly acidic, and the mixture was extracted with ethyl acetate several times. The combined organic phases were dried with sodium sulfate and the solvent was evaporated. Column chromatography (silica gel; ethyl acetate/hexane 3:1) afforded 1.05 g (5.9 mmol; 70\%) 2(2-hydroxy-5-nitrophenyl)acetonitrile. ${ }^{1} \mathrm{H}-\mathrm{NMR} \quad$ (270 MHz, acetone-d6): 8.28 (d, J=2.5Hz, $\left.1 \mathrm{H}\right), 8.11$ (dd, $\left.J_{1}=9.2 \mathrm{~Hz}, J_{2}=2.1 \mathrm{~Hz}, 1 \mathrm{H}\right), 7.15(\mathrm{~d}, J=9.2 \mathrm{~Hz}, 1 \mathrm{H}), 3.93(\mathrm{~s}, 2 \mathrm{H})$

In a flask equipped with inert gas inlet and a reflux condenser attached to a bubble counter, $1.75 \mathrm{~g}$ (9.8 mmol) 2-(2-hydroxy-5-nitrophenyl)acetonitrile was suspended in an aqueous sodium hydroxide solution 
$(10 \% \mathrm{w} / \mathrm{w})$ and refluxed $\left(120^{\circ} \mathrm{C}\right.$ bath temperature) until evolution of ammonia ceased (10 hours). The solution was acidified and the mixture was extracted with ethyl acetate several times. The combined organic phases were dried with sodium sulfate and the solvent was evaporated. Column chromatography (silica gel; dichloromethane/methanol 14:1 +3 vol.-\% HCOOH) afforded 1.73 g (8.8 mmol, 90\%) 2-(2-hydroxy-5nitrophenyl)acetic acid. Mp.: $158-159^{\circ} \mathrm{C}\left(\text { Lit. } 160-162{ }^{\circ} \mathrm{C}\right)^{18} .{ }^{1} \mathrm{H}$ NMR $(270 \mathrm{MHz}$, acetone-d6): $8.18(\mathrm{~d}, \mathrm{~J}=3 \mathrm{~Hz}$, $\left.{ }_{1 \mathrm{H}}\right), 8.06\left(\mathrm{dd}, J_{1}=9 \mathrm{~Hz}, J_{2}=3 \mathrm{~Hz}, 1 \mathrm{H}\right), 7.04(\mathrm{~d}, J=9 \mathrm{~Hz}, 1 \mathrm{H}), 3.76(\mathrm{~s}, 2 \mathrm{H})$.

\subsubsection{Carboxylic acid S9}

Analog to the procedure described in section 2.2.4.1 0.46 g of the quaternary ammonium salt S8 obtained according to section 2.2.2.3 (maximum amount ${ }^{19}$ of the organic component is $0.92 \mathrm{mmol}$ ) was treated with $0.06 \mathrm{~g} \mathrm{~K}^{13} \mathrm{CN}$ ( $\left.0.90 \mathrm{mmol}\right)$ and $0.10 \mathrm{~g} 18$-crown-6 $(0.38 \mathrm{mmol})$ for three hours at $120^{\circ} \mathrm{C}$. After acidic work-up and extraction with ethyl acetate, $0.40 \mathrm{~g}$ raw product formally corresponding to $1.1 \mathrm{mmol}$ of the nitrile (369.7 g/mol, 120\%) was obtained. ${ }^{1} \mathrm{H}$ NMR (250 MHz, acetone-d6): $8.14\left(\mathrm{~d}, J=3 \mathrm{~Hz}, 1 \mathrm{H}, \mathrm{CH}_{2} \mathrm{CCHC}\left(\mathrm{NO}_{2}\right) \mathrm{C} \underline{\mathrm{H}}\right)$, $8.08\left(\mathrm{~d}, J=3 \mathrm{~Hz}, 1 \mathrm{H}, \mathrm{CH}_{2} \mathrm{CC} \underline{\mathrm{HC}}\left(\mathrm{NO}_{2}\right) \mathrm{CH}\right), 7.15\left(\mathrm{~d}, J=3 \mathrm{~Hz}, 1 \mathrm{H}, \mathrm{CH}_{2} \mathrm{CCHCClC} \underline{\mathrm{H}}\right), 6.98(\mathrm{~d}, J=3 \mathrm{~Hz}, 1 \mathrm{H}$, $\left.\mathrm{CH}_{2} \mathrm{CC} \underline{\mathrm{HCClCH}}\right), 4.00\left(\mathrm{~s}, 2 \mathrm{H}, \mathrm{ArC}_{2} \mathrm{Ar}\right), 3.85\left(\mathrm{~d}, J=8 \mathrm{~Hz}, 2 \mathrm{H}, \mathrm{ArC}_{2}{ }^{13} \mathrm{CN}\right), 2.60\left(\mathrm{t}, J=7 \mathrm{~Hz}, 2 \mathrm{H}, \mathrm{C}_{2} \mathrm{CH}_{2} \mathrm{CH}_{3}\right)$, $1.59\left(\mathrm{qt}, J_{1}=7 \mathrm{~Hz}, J_{2}=7 \mathrm{~Hz}, 2 \mathrm{H}, \mathrm{CH}_{2} \mathrm{CH}_{2} \mathrm{CH}_{3}\right), 0.93\left(\mathrm{t}, J=7 \mathrm{~Hz}, 3 \mathrm{H}, \mathrm{CH}_{2} \mathrm{CH}_{2} \mathrm{CH}_{3}\right)$.

The nitrile was hydrolyzed by refluxing for three hours in $10 \mathrm{~mL}$ of $12.5 \%$ aqueous $\mathrm{NaOH}$. After acidification and extraction with ethyl acetate, as described in section 2.2.4.1, 0.40 g of raw product was obtained. The raw product was dissolved in $10 \mathrm{~mL}$ dichloromethane and $3 \mathrm{~mL}$ hexane was added (avoiding immediate precipitation). The solution was left over night at $-15^{\circ} \mathrm{C}$. $0.11 \mathrm{~g}$ of crystals of the acid S9 were collected by filtration. ${ }^{1} \mathrm{H} \quad \mathrm{NMR} \quad\left(250 \mathrm{MHz}, \quad\right.$ acetone-d6): 8.05-8.03 $\quad\left(2 \mathrm{H}, \quad \mathrm{C} \underline{\mathrm{HC}}\left(\mathrm{NO}_{2}\right) \mathrm{C} \underline{\mathrm{H}}\right), \quad 7.14 \quad(\mathrm{~d}, \quad J=3 \mathrm{~Hz}, \quad 1 \mathrm{H}$, $\left.\mathrm{CH}_{2} \mathrm{CCHCClC} \underline{\mathrm{H}}\right), 7.03\left(\mathrm{~d}, J=3 \mathrm{~Hz},{ }_{1} \mathrm{H}, \mathrm{CH}_{2} \mathrm{CC} \underline{\mathrm{HCClCH}}\right), 4.06\left(\mathrm{~s}, 2 \mathrm{H}, \mathrm{ArC}_{2} \mathrm{Ar}\right), 3.80(\mathrm{~d}, J=8 \mathrm{~Hz}, 2 \mathrm{H}$, $\left.\mathrm{ArC}_{2}{ }_{2}^{13} \mathrm{COOH}\right), 2.63\left(\mathrm{t}, J=7 \mathrm{~Hz}, 2 \mathrm{H}, \mathrm{C}_{2} \mathrm{CH}_{2} \mathrm{CH}_{3}\right), 1.61$ (qt, $\left.J_{1}=7 \mathrm{~Hz}, J_{2}=7 \mathrm{~Hz}, 2 \mathrm{H}, \mathrm{CH}_{2} \mathrm{CH}_{2} \mathrm{CH}_{3}\right), 0.94(\mathrm{t}$, $\left.J=7 \mathrm{~Hz}, 3 \mathrm{H}, \mathrm{CH}_{2} \mathrm{CH}_{2} \underline{\mathrm{C}}_{3}\right)$.

\subsubsection{Carboxylic acid $\mathbf{S 1 2}$}

Analog to the procedure described in section 2.2.4.1, $1.10 \mathrm{~g}(2.35 \mathrm{mmol})$ of iodide $\mathbf{S 1 1}$ was treated with $0.14 \mathrm{~g}$ $\mathrm{KOH}$ (1 eq.), o.17g KCN (1 eq.) and o.09g 18-crown-6 (o.15 eq.) for 6 hours at gradually increasing temperatures between $70-110^{\circ} \mathrm{C}$. The raw product was $0.95 \mathrm{~g}$ of brown oil. After purification by column chromatography (silica gel, ethyl acetate, $\left.R_{\mathrm{f}}=0.8\right) 0.50 \mathrm{~g}(308 \mathrm{~g} / \mathrm{mol}, 1.6 \mathrm{mmol}, 68 \%)$ of the nitrile intermediate was obtained. ${ }^{1} \mathrm{H}$ NMR $\left(270 \mathrm{MHz}, \mathrm{CDCl}_{3}\right): 7.64(\mathrm{~s}, 1 \mathrm{H}, \mathrm{ArO} \underline{\mathrm{H}}), 7.22(\mathrm{~d}, J=3 \mathrm{~Hz}, 1 \mathrm{H}, \mathrm{Ar}), 7.20(\mathrm{~d}$, 
$J=3 \mathrm{~Hz}, 1 \mathrm{H}, \mathrm{Ar}), 7.15(\mathrm{~d}, J=3 \mathrm{~Hz}, 1 \mathrm{H}, \mathrm{Ar}), 7.05\left(\mathrm{dd}, J_{1}=9 \mathrm{~Hz}, J_{2}=3 \mathrm{~Hz},{ }_{1} \mathrm{H}, \mathrm{CHC} \underline{\mathrm{HCClCH}}\right), 6.70\left(\mathrm{~d}, J=9 \mathrm{~Hz},{ }_{1} \mathrm{H}\right.$, $\mathrm{C} \underline{\mathrm{HCHCClCH}}), 6.20(\mathrm{~s}, 1 \mathrm{H}, \mathrm{ArO} \underline{\mathrm{H}}), 3.81$ (s, 2H, $\left.\mathrm{ArC} \underline{\mathrm{H}}_{2} \mathrm{Ar}\right), 3.64\left(\mathrm{~s}, 2 \mathrm{H}, \mathrm{ArC}_{2} \mathrm{CN}\right)$.

The nitrile was hydrolyzed analog to the description in 2.2.4.1. Chromatographic isolation of the carboxylic acid was done with silica gel and ethyl acetate/hexane $2: 1+4 \%$ formic acid as eluent.

$0.34 \mathrm{~g}$ (327 g/mol, $1.0 \mathrm{mmol}, 63 \%)$ of the carboxylic acid $\mathbf{S 1 2}$ was obtained. ${ }^{1} \mathrm{H}$ NMR (270 MHz, acetone-d6): 9.52 (s (broad), $2 \mathrm{H}, \mathrm{O} \underline{\mathrm{H}}), 7.23(\mathrm{~d}, J=3 \mathrm{~Hz}, 1 \mathrm{H}, \mathrm{Ar}), 7.15$ (d, J= $3 \mathrm{~Hz}, 1 \mathrm{H}, \mathrm{Ar}), 7.13(\mathrm{~d}, J=3 \mathrm{~Hz}, 1 \mathrm{H}, \mathrm{Ar}), 7.09$ (dd, $\left.J_{1}=9 \mathrm{~Hz}, J_{2}=3 \mathrm{~Hz}, 1 \mathrm{H}, \mathrm{CHC} \underline{\mathrm{HCClCH}}\right), 6.91(\mathrm{~d}, J=9 \mathrm{~Hz}, 1 \mathrm{H}, \mathrm{C} \underline{\mathrm{HCHCClCH}}), 3.95\left(\mathrm{~s}, 2 \mathrm{H}, \mathrm{ArC}_{2} \mathrm{Ar}\right), 3.69(\mathrm{~s}, 2 \mathrm{H}$, $\left.\mathrm{ArC} \underline{\mathrm{H}}_{2} \mathrm{COOH}\right)$.

\subsubsection{Carboxylic acid S16}

$21.5 \mathrm{~g}$ tetramethylammonium iodide $\mathbf{S}_{15}(500.38 \mathrm{~g} / \mathrm{mol}, 43 \mathrm{mmol}), 5.0 \mathrm{~g} \mathrm{KCN}(65 \mathrm{~g} / \mathrm{mol}, 75 \mathrm{mmol}), 2.0 \mathrm{~g}$ $\mathrm{KOH}(56 \mathrm{~g} / \mathrm{mol}, 36 \mathrm{mmol})$ and $2.0 \mathrm{~g}$ 18-crown-6 (ca. $9 \mathrm{mmol}$ ) were heated to $90^{\circ} \mathrm{C}$ in $40 \mathrm{~mL}$ DMF for nine hours. After removal of the solvent in high vacuum, water was added and $\mathrm{pH}$ was adjusted to slightly acidic. The product was extracted with ethyl acetate. The raw product was filtered through silica gel with ethyl acetate/hexane 1:1 and $2 \%$ formic acid as solvent. $10.0 \mathrm{~g}$ of the nitrile $(340.4 \mathrm{~g} / \mathrm{mol}, 29.4 \mathrm{mmol}, 68 \%)$ was obtained. ${ }^{1} \mathrm{H}$ NMR $\left(270 \mathrm{MHz}\right.$, acetone- $\left.\mathrm{d}_{6}\right): 8.03\left(\mathrm{~s}, 1 \mathrm{H}, \mathrm{CHCHC}\left(\mathrm{NO}_{2}\right) \mathrm{C} \underline{\mathrm{H}}\right), 8.01\left(\mathrm{~d}, J=9 \mathrm{~Hz}, \mathrm{CHC} \underline{\mathrm{HC}}\left(\mathrm{NO}_{2}\right) \mathrm{CH}\right)$, 7.10 (s, $2 \mathrm{H}, \mathrm{C} \underline{\mathrm{HC}}(\mathrm{s}-\mathrm{Bu}) \mathrm{C} \underline{\mathrm{H}}), 7.08\left(\mathrm{~d}, J=9 \mathrm{~Hz}, \mathrm{C} \underline{\mathrm{HCHC}}\left(\mathrm{NO}_{2}\right) \mathrm{CH}\right), 4.07\left(\mathrm{~s}, 2 \mathrm{H}, \mathrm{ArC}_{2} \mathrm{Ar}\right), 3.82\left(\mathrm{~s}, 2 \mathrm{H}, \mathrm{ArC} \underline{\mathrm{H}}_{2} \mathrm{CN}\right)$, $2.52\left(\mathrm{qt}, J_{1}=J_{2}=7 \mathrm{~Hz}, 1 \mathrm{H}, \mathrm{CH}_{3} \underline{\mathrm{HCH}}_{2}\right), 1.53\left(\mathrm{dq}, J_{1}=J_{2}=7 \mathrm{~Hz}, 2 \mathrm{H}, \mathrm{CHC} \underline{\mathrm{H}}_{2} \mathrm{CH}_{3}\right), 1.17\left(\mathrm{~d}, J=7 \mathrm{~Hz}, 3 \mathrm{H}, \mathrm{CHC}_{3}\right)$, $0.77\left(\mathrm{t}, J=7 \mathrm{~Hz}, 3 \mathrm{H}, \mathrm{CH}_{2} \underline{\mathrm{C}}_{3}\right)$.

The nitrile was suspended in $35 \mathrm{~mL} \mathrm{10 \%} \mathrm{KOH}$ and refluxed for 24 hours. The solution was made acidic and extracted with ethyl acetate. The organic phase was dried, and the solvent was evaporated. The raw product was filtered through silica gel (solvent ethyl acetate and $2 \%$ formic acid). The filtrate contained $8.5 \mathrm{~g}$ of substance. The product was isolated by column chromatography (silica gel, dichloromethane/methanol 10:1 to 5:1). $7.5 \mathrm{~g}$ carboxylic acid S16 (359.4 g/mol, 20.9 mmol, 71 \%) was obtained. ${ }^{1} \mathrm{H}$ NMR (50o MHz, acetone-d6): $8.03\left(\mathrm{~d}, J=3 \mathrm{~Hz}, 1 \mathrm{H}, \mathrm{CHCHC}\left(\mathrm{NO}_{2}\right) \mathrm{C} \underline{\mathrm{H}}\right), 7.99\left(\mathrm{dd}, J_{1}=9 \mathrm{~Hz}, J_{2}=3 \mathrm{~Hz}, \mathrm{CHC} \underline{\mathrm{HC}}\left(\mathrm{NO}_{2}\right) \mathrm{CH}\right), 7.03(\mathrm{~d}, J=9 \mathrm{~Hz}$, C$\left.\underline{\mathrm{HCHC}}\left(\mathrm{NO}_{2}\right) \mathrm{CH}\right), 7.03(\mathrm{~s}, 2 \mathrm{H}, \mathrm{CHC}(\mathrm{s}-\mathrm{Bu}) \mathrm{CH}), 6.95(\mathrm{~s}, 2 \mathrm{H}, \mathrm{CHC}(s-\mathrm{Bu}) \mathrm{CH}), 4.05\left(\mathrm{~s}, 2 \mathrm{H}, \mathrm{ArC}_{2} \mathrm{Ar}\right), 3.71(\mathrm{~s}, 2 \mathrm{H}$, $\left.\mathrm{ArC} \underline{\mathrm{H}}_{2} \mathrm{COOH}\right), 2.49\left(\mathrm{tq}, J_{1}=J_{2}=7 \mathrm{~Hz},{ }_{1} \mathrm{H}, \mathrm{CH}_{3} \mathrm{CHCH}_{2}\right), 1.52\left(\mathrm{qd}, J_{1}=J_{2}=7 \mathrm{~Hz}, 2 \mathrm{H}, \mathrm{CHC}_{2} \mathrm{CH}_{3}\right), 1.16(\mathrm{~d}, J=7 \mathrm{~Hz}$, $\left.{ }_{3} \mathrm{H}, \mathrm{CHC} \underline{\mathrm{H}}_{3}\right), 0.78\left(\mathrm{t}, J=7 \mathrm{~Hz}, 3 \mathrm{H}, \mathrm{CH}_{2} \underline{\mathrm{C}}_{3}\right)$. 


\subsubsection{Carboxylic acid S19}

4.o $\mathrm{g}$ of the quaternary ammonium salt $\mathrm{S} 18$ obtained according to section 2.2.2.5 (maximum amount ${ }^{17}$ of the organic component was $6.0 \mathrm{mmol}$ ) was dissolved in $40 \mathrm{~mL}$ DMF. $1.0 \mathrm{~g} \mathrm{KCN} \mathrm{(2.5} \mathrm{eq.)} \mathrm{and} \mathrm{1.o} \mathrm{g} \mathrm{18-crown-6} \mathrm{(ca.}$ o.6 eq.) were added. The mixture was stirred and heated to $90^{\circ} \mathrm{C}-110^{\circ} \mathrm{C}$ for 18 hours until the evolution of ammonia had ceased. DMF was removed by distillation in high vacuum followed by an acidic aqueous workup and extraction with ethyl acetate. $4.4 \mathrm{~g}$ of a brown oil was obtained. This was dissolved in ethyl acetate and filtered through silica gel. After evaporation of the solvent, $1.6 \mathrm{~g}$ of a golden colored foam remained.

The nitrile (1.6 g) was hydrolyzed by refluxing in $20 \% \mathrm{NaOH}$ until evolution of ammonia had ceased (12 hours). The solution was made acidic and extracted with acetyl acetate. The raw product was subjected to column chromatography (silica gel, ethyl acetate /hexane $1: 1$ with $2 \%$ formic acid). o.8o g of the carboxylic acid $\mathbf{S 1 9}$ was isolated. ${ }^{1} \mathrm{H}$ NMR (5oo MHz, acetone-d6): $8.15\left(\mathrm{~d}, J=3 \mathrm{~Hz},{ }_{1} \mathrm{H}, \mathrm{CHC}\left(\mathrm{NO}_{2}\right) \mathrm{CH}\right), 8.04(\mathrm{~d}, J=3 \mathrm{~Hz}$, $\left.{ }_{1} \mathrm{H}, \mathrm{CHC}\left(\mathrm{NO}_{2}\right) \mathrm{CH}\right), 7.15(\mathrm{~d}, J=3 \mathrm{~Hz}, 1 \mathrm{H}, \mathrm{CHC}(\mathrm{s}-\mathrm{Bu}) \mathrm{CH}), 6.87(\mathrm{~d}, J=3 \mathrm{~Hz}, 1 \mathrm{H}, \mathrm{CHC}(\mathrm{s}-\mathrm{Bu}) \mathrm{CH}), 4.05(\mathrm{~s}, 2 \mathrm{H}$, $\left.\mathrm{ArCH}_{2} \mathrm{Ar}\right), 3.94\left(\mathrm{t}, J=6 \mathrm{~Hz}, 2 \mathrm{H}, \mathrm{CH}_{2} \mathrm{CH}_{2} \mathrm{OH}\right), 3.76\left(\mathrm{~s}, 2 \mathrm{H}, \mathrm{ArCH}_{2} \mathrm{COOH}\right), 2.90\left(\mathrm{t}, J=6 \mathrm{~Hz}, 2 \mathrm{H}, \mathrm{CH}_{2} \mathrm{CH}_{2} \mathrm{OH}\right)$, $2.49\left(\mathrm{qt}, J_{1}=J_{2}=7 \mathrm{~Hz}, 1 \mathrm{H}, \mathrm{CH}_{3} \underline{\mathrm{HCH}}_{2}\right), 1.53\left(\mathrm{dq}, J_{1}=J_{2}=7 \mathrm{~Hz}, 2 \mathrm{H}, \mathrm{CHCH}_{2} \mathrm{CH}_{3}\right), 1.15\left(\mathrm{~d}, J=7 \mathrm{~Hz},{ }_{3} \mathrm{H}, \mathrm{CHC} \underline{\mathrm{H}}_{3}\right)$, $0.77\left(\mathrm{t}, J=7 \mathrm{~Hz},{ }_{3} \mathrm{H}, \mathrm{CH}_{2} \underline{\mathrm{CH}}_{3}\right)$.

\subsubsection{2-(2-hydroxyphenyl)-ethanol derivatives}

\subsubsection{Reduction of the carboxylic acid S16 to alcohol}

$5.50 \mathrm{~g}$ of the carboxylic acid S16 $(359.4 \mathrm{~g} / \mathrm{mol} ; 15.3 \mathrm{mmol})$ was dissolved in $15 \mathrm{~mL}$ of dry THF. $14 \mathrm{~mL}$ of a $2 \mathrm{M}$ solution of $\mathrm{BH}_{3} \mathrm{~S}\left(\mathrm{CH}_{3}\right)_{2}(28 \mathrm{mmol})$ was added dropwise. Gas evolution was observed. Conversion of the acid $\left(R_{\mathrm{f}}=0.5\right)$ to the alcohol $\left(R_{\mathrm{f}}=0.8\right)$ and a side product $\left(R_{\mathrm{f}}=0.9\right)$ was monitored by TLC (silica gel; dichloromethane/methanol 10:1). The solution was stirred over night at room temperature. Water was added carefully. The solution was acidified ( $\mathrm{pH} \mathrm{1-4}$ ) and extracted with ethyl acetate. The organic phase was dried with sodium sulfate and the solvent was evaporated. The product was separated from the less polar side product by column chromatography (silica gel; ethyl acetate /hexane 1:1): $4.58 \mathrm{~g}$ (345.4 g/mol; $13.3 \mathrm{mmol}$; 86\%). ${ }^{1} \mathrm{H}$ NMR (25o MHz, acetone-d6 ): $8.05\left(\mathrm{~d}, J=3 \mathrm{~Hz},{ }_{1} \mathrm{H}, \mathrm{CHCHC}\left(\mathrm{NO}_{2}\right) \mathrm{C} \underline{\mathrm{H}}\right), 7.96\left(\mathrm{dd}, J_{1}=9 \mathrm{~Hz}, J_{2}=3 \mathrm{~Hz}\right.$, $\left.1 \mathrm{H}, \mathrm{CHC} \underline{\mathrm{HC}}\left(\mathrm{NO}_{2}\right) \mathrm{CH}\right), 7.01(\mathrm{~s}, 1 \mathrm{H}, \mathrm{CHC}(s-\mathrm{Bu}) \mathrm{CHCCl}), 6.98\left(\mathrm{~d}, J=9 \mathrm{~Hz}, 1 \mathrm{H}, \mathrm{C} \underline{\mathrm{HCHC}}\left(\mathrm{NO}_{2}\right) \mathrm{CH}\right), 6.84(\mathrm{~s}, 1 \mathrm{H}$, $\mathrm{CHC}(s-\mathrm{Bu}) \mathrm{CHCCl}), 4.01\left(\mathrm{~s}, \quad 2 \mathrm{H}, \quad \mathrm{ArCH}_{2} \mathrm{Ar}\right), 3.90\left(\mathrm{t}, J=6 \mathrm{~Hz}, 2 \mathrm{H}, \mathrm{CH}_{2} \mathrm{CH}_{2} \mathrm{OH}\right), \quad 2.86 \quad(\mathrm{t}, J=7 \mathrm{~Hz}, 2 \mathrm{H}$, 
$\left.\mathrm{CH}_{2} \mathrm{CH}_{2} \mathrm{OH}\right), 2.45\left(\mathrm{qt}, J_{1}=J_{2}=7 \mathrm{~Hz}, 1 \mathrm{H}, \mathrm{CH}_{3} \mathrm{CHCH}_{2}\right), 1.51\left(\mathrm{dq}, J_{1}=J_{2}=7 \mathrm{~Hz}, 2 \mathrm{H}, \mathrm{CHCH}_{2} \mathrm{CH}_{3}\right), 1.14(\mathrm{~d}, J=7 \mathrm{~Hz}$, $\left.3 \mathrm{H}, \mathrm{CHCH}_{3}\right), 0.76\left(\mathrm{t}, \mathrm{J}=7 \mathrm{~Hz}, 3 \mathrm{H}, \mathrm{CH}_{2} \mathrm{C}_{3}\right)$. 


\subsection{Spectroscopy}

\subsubsection{Instrumentation, sample preparation}

The equipment and general procedures for combined NMR and UV-vis spectroscopy were previously described in Refs. 20.

Solutions of monoanions were prepared from the neutral compounds by addition of a slight excess of tetraethylammonium hydroxide solution in methanol. Solvent and water were removed in vacuum (10 $\mathrm{mL}$ flask, rotary evaporator, < 10 mbar residual pressure). Further drying was achieved by repeated addition and evaporation of dry $\mathrm{CH}_{2} \mathrm{Cl}_{2}$.under reduced pressure (rotavap $<10 \mathrm{mbar}$ ). The residue was taken up in $\mathrm{CD}_{2} \mathrm{Cl}_{2}$ and transferred to a flat bottomed quartz tube (“UVNMR cuvette”, see Ref. 30) with PTFE insert for combined NMR and UV-vis spectroscopy. In cases of evidence for the presence of di-anionic species (according to any part of the combined spectroscopy), split equivalent amounts of the corresponding neutral compound were added as a solution in $\mathrm{CD}_{2} \mathrm{Cl}_{2}$ until the monoanion was present exclusively (a titration guided by NMR or the combined spectroscopy, see Refs. 20).

For subsequent experiments in $\mathrm{CDF}_{2} \mathrm{Cl} / \mathrm{CDF}_{3}$ as solvent, sample solutions were transferred to medium wall sized NMR tubes equipped with $J$. Young valves and PTFE inserts. After evaporation of $\mathrm{CD}_{2} \mathrm{Cl}_{2}$ in vacuum, $\mathrm{CDF}_{2} \mathrm{Cl} / \mathrm{CDF}_{3}$ was added by vacuum transfer.

Partial deuteration in mobile proton sites was generally done in NMR tubes equipped with J. Young valves. From a sample solution prepared as described above, the solvent was removed and MeOD (o.1 mL) was added to the residual sample substance. Methanol was removed again under reduced pressure and the solvent desired for spectroscopic studies was reintroduced.

Solute concentrations typically were $1 \mathrm{mM}$ for the combined spectroscopy and up to $3 \mathrm{mM}$ if only NMR spectra were to be obtained. For the former, concentrations were practically limited by optical density. For the latter, increase in concentration was principally motivated by sensitivity concerns but limitations arose from issues of solubility (solute-solute interactions). 


\subsubsection{Evaluation}

${ }^{1} \mathrm{H}$ and ${ }^{13} \mathrm{C}$ NMR chemical shifts were determined using solvent signals as internal standard, and converted to the conventional TMS scale: $\delta\left(\mathrm{CHDCl}_{2}\right)=5.32 \mathrm{ppm}, \quad \delta\left({ }^{13} \mathrm{CD}_{2} \mathrm{Cl}_{2}\right)=53.5 \mathrm{ppm}, \quad \delta\left(\mathrm{CHF}_{2} \mathrm{Cl}\right)=7.18 \mathrm{ppm}$, $\delta\left({ }^{13} \mathrm{CDF}_{2} \mathrm{Cl}\right)=117.37 \mathrm{ppm}$. Proton decoupled ${ }^{13} \mathrm{C}$ NMR spectra were obtained using the inverse-gated decoupling scheme allowing us to employ signal integration in the attribution to different isotopologues and their isotopomers (if any).

In systems with coupled $\mathrm{H}$-bonds, integral signal intensities of the isotopologues/isotopomers were analyzed in order to assess isotope fractionation effects. ${ }^{1} \mathrm{H}$ NMR, by nature, can give information only on species with at least one hydrogen atom in the H-bond chain (if only the H-bonding protons are considered). While ${ }^{13} \mathrm{C}$ NMR, e.g. of the carboxylic carbon, can provide that information for all species whose individual signal contributions are resolved, spectra tend to suffer from low signal to noise ratios - or are not available at all, namely for species with natural carbon isotope abundance.

The information gap on the fully deuterated species that ${ }^{1} \mathrm{H}$ NMR leaves can be filled if one assumes, in an approximation, that the nature of the light particle in one binding site does not affect the potential of the particles in the other sites of the H-bond chain (negligence of the vicinal isotope effect). This simplification seems justified because in all the systems studied in this work, the vicinal isotope effects (in terms of chemical shifts) are at least an order of magnitude smaller than the shifts between signals of the protons in the individual binding sites. Neglecting the vicinal isotope means that the equilibrium constants of the following two exchange reactions (in which e.g. "HH" identifies a system of two coupled H-bonds containing only protons as light particles) are identical:

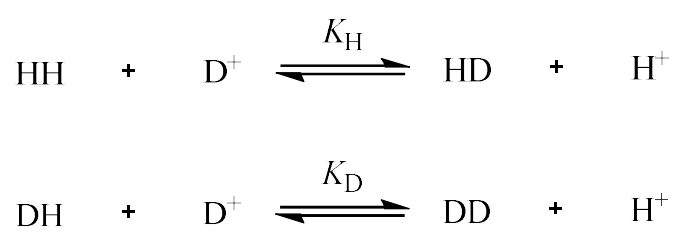

For this situation, it can readily be deduced from the chemical equilibrium law that the concentration of the fully deuterated species is (within the limitations of the above approximation):

$c(\mathrm{DD}) \approx c(\mathrm{HD}) c(\mathrm{DH}) / c(\mathrm{HH})$

which corresponds to: 
$\mathrm{HH}+\mathrm{DD} \stackrel{K=1}{\rightleftharpoons} \mathrm{HD}+\mathrm{DH}$

Analogue relationships can be established also for systems with three coupled H-bonds. Thus, (relative) concentrations of isotopologues/isotopomers (Table S 8) were established from integral intensities of proton as well as carbon spectra (Table $S_{7}$ ). Whenever redundant information was present in the spectra (e.g. the intensity of the two proton signals in a system of the type $\mathrm{HH}$ ), averaged values were used for evaluation. Furthermore, deuterium fractions in individual light particle sites for isotopologues/isotopomers and isotope fractionation factors were calculated wherever possible (Table S 9). The site specific deuterium fractions are defined as follows, for the example of the bond to the carboxylic group in $\mathbf{4} \mathbf{b}$ :

$x_{\underline{\mathrm{DLL}}}=c_{\underline{\mathrm{DLL}}} /\left(c_{\underline{\mathrm{HLL}}}+c_{\underline{\mathrm{DLL}}}\right)$

where the concentrations containing the light particle wildcard $\mathrm{L}=\mathrm{H}$ or $\mathrm{D}$ expand to, e.g.:

$c_{\underline{\mathrm{DLL}}}=c_{\underline{\mathrm{DHH}}}+c_{\underline{\mathrm{DHD}}}+c_{\underline{\mathrm{DDH}}}+c_{\underline{\mathrm{DDD}}}$

and (relative) values for the concentrations $c$ are derived from integral signal intensities. 


\section{Results}

3.1.1 UV-vis absorption spectra of 4-nitrophenols, their anions and homoconjugated anions

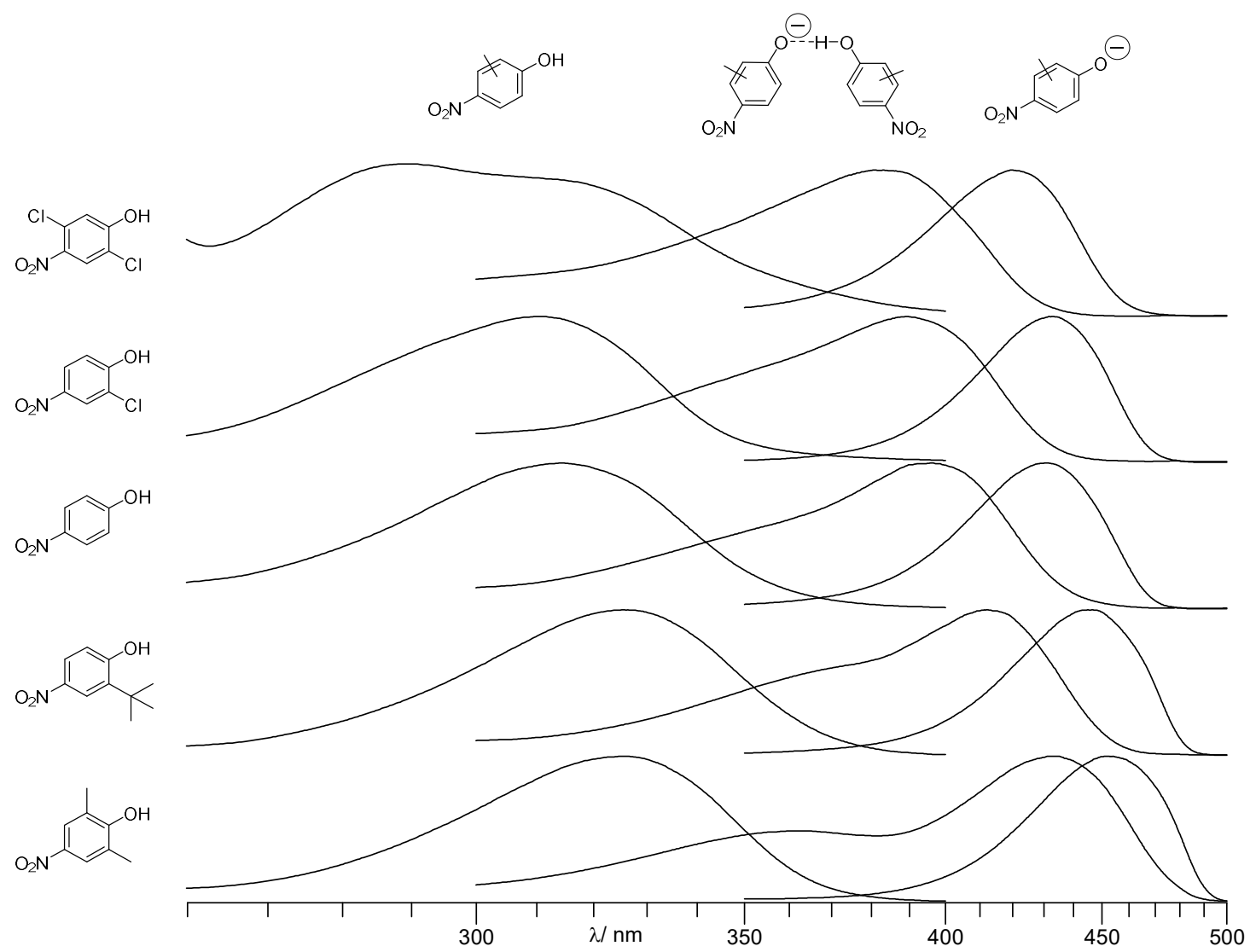

Figure S 6. Absorption spectra of the $\pi-\pi^{*}$ transition of 4-nitrophenols, their anions and homoconjugated anions in $\mathrm{CD}_{2} \mathrm{Cl}_{2}$ solution at $170-18 \mathrm{o}$ K. Counter ions are tetraethylammonium. All spectra were normalized to equal maxima. For clarity, traces were reduced to the essential spectral regions. Note how the dual band character of homoconjugated anions seems to become particular obvious in the last two (sterically hindered) species. 


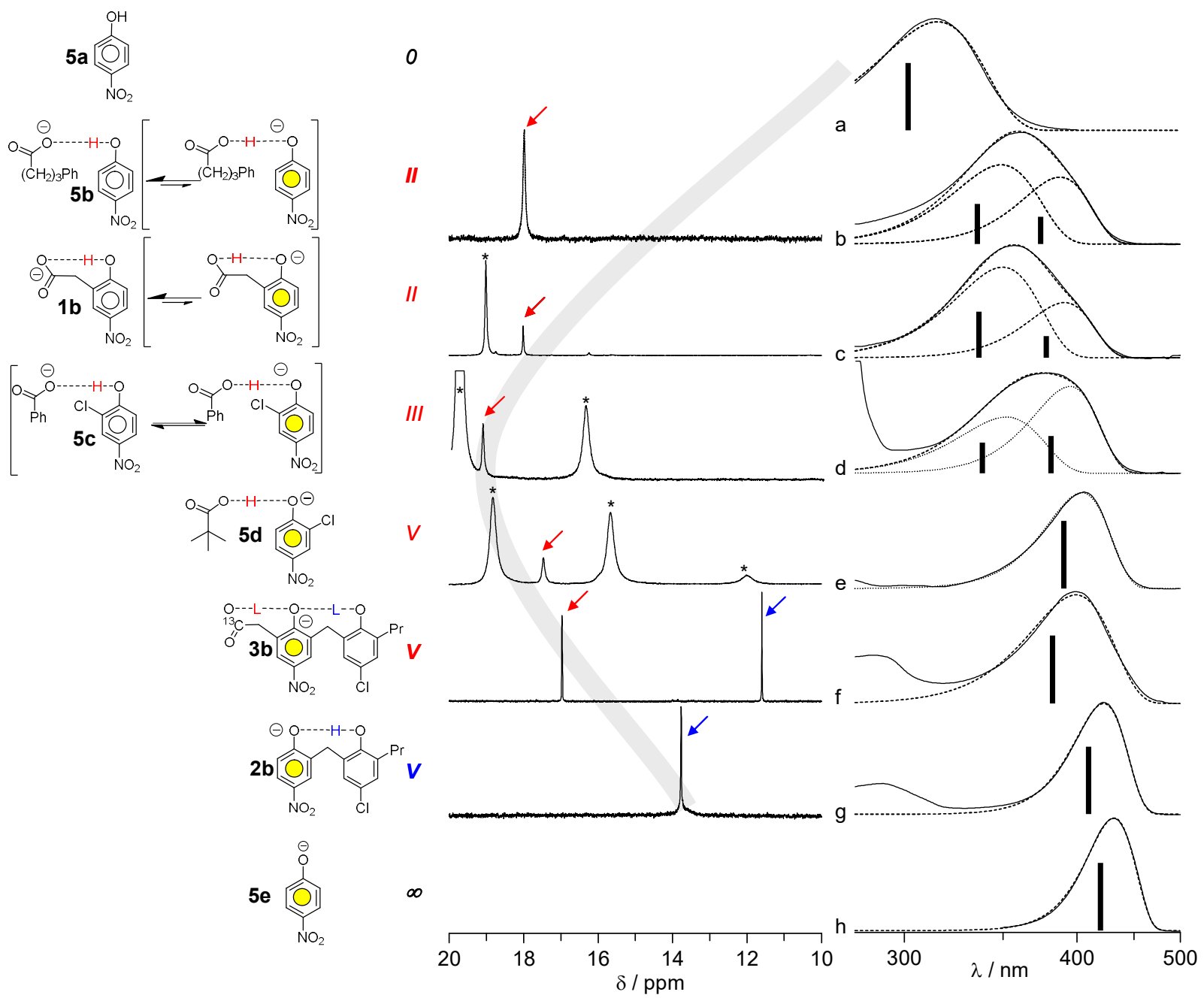

Figure $\mathrm{S}_{7}$. UVNMR spectra (simultaneously obtained ${ }^{1} \mathrm{H}$ NMR and UV-vis absorption spectra of $\mathrm{CD}_{2} \mathrm{Cl}_{2}$ solution at $180 \mathrm{~K}$ ) of anions with intramolecular hydrogen bonds (studied in this work) and reference systems 5 (Refs. 20 and 21). The same conventions apply as in Figure 3 of the main text. Diagonal arrows indicate NMR signals that originate from protons in the respective chem. structures and the roman numbers characterize H-bonds according to Figure 4 (3b: left proton $\rightarrow$ left signal; characterization of H-bond to carboxylic group). The eight systems are given in the sequence of progressive deprotonation (increasing anionic character) of the nitrophenolic moieties in the fashion of Figure 4 , as evidenced by the red-shift in light absorption and the strong deshielding of the bridging protons culminating in stage III (light gray arc in the background is a rough guide for the eye). The minor discontinuity among the optical spectra of $\mathbf{5} \mathbf{d}$ and $\mathbf{3} \mathbf{b}$ could be a direct spectral effect of the ring substituents of the phenolic moieties (see Figure S 6: the spectra of neutral phenols and "free phenolates" display some spectral variations on ring substitution.). 


\subsection{Tables of spectroscopic data}

Table $S_{3}$. UV-vis spectroscopic parameters of monoanions $1 b-3 b$, and $4 b$ at various conditions.

\begin{tabular}{|l|l|l|l|l|l|l|l|l|l|l|}
\hline species & solvent & $T$ & $\lambda_{\max }$ & $\tilde{v}_{\text {COG }}$ & $\Delta \tilde{v}$ & $\tilde{v}_{\text {blue }}$ & $\Delta \tilde{v}_{\text {blue }}$ & $\tilde{v}_{\text {red }}$ & $\Delta \tilde{v}_{\text {red }}$ & $x_{\text {blue }}$ \\
\hline $\mathbf{1 b}$ & $\mathrm{CD}_{2} \mathrm{Cl}_{2}$ & 180 & 360 & 28550 & 6180 & 29800 & 5030 & 26750 & 4070 & 0.59 \\
\hline $\mathbf{2 b}$ & $\mathrm{CD}_{2} \mathrm{Cl}_{2}$ & 180 & 422 & 24410 & 3090 & & & & & 0 \\
\hline $\mathbf{3 b}$ & $\mathrm{CD}_{2} \mathrm{Cl}_{2}$ & 180 & 400 & 25900 & 4500 & & & & & 0 \\
\hline $\mathbf{3 b}$ & $\mathrm{CDF}_{2} \mathrm{Cl} / \mathrm{CDF}_{3}$ & 130 & 404 & 26200 & 5910 & & & & & 0 \\
\hline $\mathbf{4 b}$ & $\mathrm{CD}_{2} \mathrm{Cl}_{2}$ & 210 & 391 & 26900 & 5710 & & & & & 0 \\
\hline
\end{tabular}

Temperatures $T$ are given in Kelvin, wavenumbers $\tilde{v}$ in $\mathrm{cm}^{-1}$ and wavelength $\lambda$ in $\mathrm{nm}$. Parameters of UV-vis absorption bands are center of gravity $\tilde{v}_{\mathrm{COG}}$, half height full width $\Delta \widetilde{v}$ and wavelength of maximum in absorbance $\lambda_{\max }$. Results of deconvolution in terms of dual bands are given as center of gravities $\tilde{v}_{\text {blue }}$ and $\tilde{v}_{\text {red }}$ and half height widths $\Delta \tilde{v}_{\text {blue }}$ and $\Delta \tilde{v}_{\text {red }}$ where the indices denote the components at higher and lower energies, respectively. Relative intensities of the components are given as fraction of integral absorbance $E$ of the higher energy component $x_{\text {blue }}=\frac{E_{\text {blue }}}{E_{\text {blue }}+E_{\text {red }}}$

Table S 4. NMR spectroscopic parameters of monoanions $1 b$ and $2 b$ under various conditions.

\begin{tabular}{|l|l|l|l|l|l|}
\hline \multirow{2}{*}{ Species } & Solvent & $T$ & $\delta(\mathrm{O} \underline{\mathrm{HO}})$ & $\delta(\underline{\mathrm{COOHO}})$ & $\begin{array}{l}\delta(\underline{\mathrm{COODO}})- \\
\delta(\underline{\mathrm{COOHO}})\end{array}$ \\
\hline \multirow{3}{*}{$\mathbf{b}$} & $\mathrm{CD}_{2} \mathrm{Cl}_{2}$ & 180 & 18.09 & 175.31 & \\
\cline { 3 - 7 } & & 170 & 17.99 & & \\
\cline { 2 - 6 } & $\mathrm{CDF}_{2} \mathrm{Cl} / \mathrm{CDF}_{3}$ & 170 & 17.32 & 175.07 & -0.075 \\
\cline { 3 - 6 } & & 120 & 17.22 & 175.40 & -0.058 \\
\hline $2 \mathbf{b}$ & $\mathrm{CD}_{2} \mathrm{Cl}_{2}$ & 180 & 13.77 & & \\
\hline
\end{tabular}

Temperatures $T$ are given in Kelvin, chemical shifts $\delta$ in ppm. For the attribution of chemical shifts, molecular fragments are given in shorthand notation and the observed nucleus is underlined. $L=H$ or $D$.

Table $S_{5}$. NMR spectroscopic parameters of monoanions $3 b$ and $3 c$ under various conditions.

\begin{tabular}{|c|c|c|c|c|c|c|c|c|c|c|}
\hline \multirow{2}{*}{ Species } & \multirow{2}{*}{ Solvent } & \multirow{2}{*}{$T$} & \multicolumn{4}{|c|}{$\delta($ COOLOLO $)$} & \multicolumn{4}{|c|}{$\delta(\underline{\text { COOLOLO })}$} \\
\hline & & & $\underline{\mathrm{H}} \mathrm{H}$ & HD & $\mathrm{H} \underline{\mathrm{H}}$ & $\mathrm{D} \underline{\mathrm{H}}$ & $\mathrm{HH}$ & HD & $\mathrm{DH}$ & DD \\
\hline \multirow[t]{2}{*}{$3 b$} & $\mathrm{CD}_{2} \mathrm{Cl}_{2}$ & 180 & 17.03 & 17.13 & 11.61 & 11.86 & 173.92 & 173.97 & 173.58 & 173.62 \\
\hline & $\mathrm{CDF}_{2} \mathrm{Cl} / \mathrm{CDF}_{3}$ & 130 & 18.13 & 18.25 & 11.72 & 12.09 & 178.09 & 178.15 & 177.60 & 177.66 \\
\hline \multirow[t]{3}{*}{$3 c$} & $\mathrm{CD}_{2} \mathrm{Cl}_{2}$ & 180 & 18.77 & & 11.37 & 11.45 & & & & \\
\hline & $\mathrm{CDF}_{2} \mathrm{Cl} / \mathrm{CDF}_{3}$ & 150 & 18.52 & & 10.93 & & & & & \\
\hline & & 140 & 18.36 & 18.27 & 10.81 & 10.44 & & & & \\
\hline
\end{tabular}

Temperatures $T$ are given in Kelvin, chemical shifts $\delta$ in ppm. For the attribution of chemical shifts, molecular fragments are given in shorthand notation and the observed nucleus is underlined. Light particle wildcard $\mathrm{L}=\mathrm{H}$ or D. The assignment of isotopologues and their isotopomers follows the syntax introduced in main text Figure 3, i.e. "HD" corresponds to a semi-deuterated species with the proton in the hydrogen bond of the carboxylic group. 
Table S 6. Mobile proton chemical shifts of monoanion 4 b under various conditions.

\begin{tabular}{|c|c|c|c|c|c|c|c|c|}
\hline \multirow{2}{*}{ Specie } & \multirow{2}{*}{ Solvent } & \multirow{2}{*}{$T$} & \multicolumn{6}{|c|}{$\delta($ COOLOLOLO $)$} \\
\hline & & & HHL & $\mathrm{H} \underline{\mathrm{H}} \mathrm{H}$ & $\mathrm{H} \underline{\mathrm{HD}}$ & $\mathrm{DHL}$ & $\mathrm{D} \underline{\mathrm{H}} \mathrm{H}$ & D \\
\hline \multirow{2}{*}{$4 b$} & $\mathrm{CD}_{2} \mathrm{Cl}_{2}$ & 210 & \begin{tabular}{|l|l|l|}
16.94 & 17.06 & 11.87 \\
\end{tabular} & & & 12.14 & & \\
\hline & $\mathrm{CDF}_{2} \mathrm{Cl} /$ & 150 & \begin{tabular}{|l|l|}
17.85 & 18.00 \\
\end{tabular} & $12.28^{\mathrm{a}}$ & $12.24^{\mathrm{a}}$ & & 12.6 & 12.69 \\
\hline
\end{tabular}

Temperatures $T$ are given in Kelvin, chemical shifts $\delta$ in ppm. For the attribution of chemical shifts, molecular fragments are given in shorthand notation and the observed nucleus is underlined. Light particle wildcard $\mathrm{L}=\mathrm{H}$ or D. The assignment of isotopologues and their isotopomers follows the syntax introduced in main text Figure 6, i.e. "DㄴL" corresponds to the signal of a proton in the phenol-phenol $\mathrm{H}$-bond of a partially deuterated species with a deuteron in the H-bond of the carboxylic group and any light particle in the aliphatic alcohol. ${ }^{\text {a }}$ Signals are asymmetric doublets due to a non-isotope related $0.035 \mathrm{ppm}$ splitting; in each case the weighted average chemical shift is given.

Table $\mathrm{S}_{7}$. Integral signal intensities $I$ in ${ }^{1} \mathrm{H}$ and ${ }^{13} \mathrm{C}$ spectra of species $3 \mathrm{~b}, 3 \mathrm{C}$ and $4 \mathrm{~b}$.

a.

\begin{tabular}{|c|c|c|c|c|c|c|c|c|c|c|c|}
\hline species & spectrum & solvent & $T$ & \multicolumn{4}{|c|}{$I(\mathrm{COOLOLO})$} & \multicolumn{4}{|c|}{$I\left({ }^{13} \mathrm{COOLOLO}\right)$} \\
\hline & & & & $\underline{\mathrm{H} H}$ & $\underline{\mathrm{HD}}$ & $\mathrm{H} \underline{\mathrm{H}}$ & $\mathrm{D} \underline{\mathrm{H}}$ & $\mathrm{HH}$ & HD & $\mathrm{DH}$ & DD \\
\hline $3 \mathbf{b}$ & Figu & $\mathrm{CD}_{2} \mathrm{Cl}_{2}$ & 185 & 28.1 & 27.9 & 27.5 & 16.5 & 35.1 & 30.3 & 17.2 & 17.4 \\
\hline $3 \mathbf{b}$ & Figure ze & $\mathrm{CDF}_{2} \mathrm{Cl} / \mathrm{CDF}_{3}$ & 130 & $27 \cdot 4$ & 9.0 & 0.9 & 12.6 & 32.9 & 39.5 & 12.6 & 14.9 \\
\hline $3 \mathbf{b}$ & Fig & $\mathrm{CDF}_{2} \mathrm{Cl} / \mathrm{CDF}_{3}$ & 130 & 35.4 & 21.3 & 34.6 & 8.7 & 47.8 & 35.0 & 11.7 & 5.5 \\
\hline 3c & Figure 5 & $\mathrm{CDF}_{2} \mathrm{Cl} / \mathrm{CDF}_{3}$ & 140 & 39.71 & 15.5 & 40.32 & 4.4 & n.d. & n.d. & n.d. & n.d. \\
\hline
\end{tabular}

b.

\begin{tabular}{|c|c|c|c|c|c|c|c|c|c|c|c|}
\hline species & spectrum & solvent & $T$ & $\mathrm{I}(\mathrm{COC}$ & OLOL & & & & & & \\
\hline & & & & $\underline{\mathrm{HHL}}$ & $\underline{\mathrm{HDL}}$ & HㅁL & $\mathrm{H} \underline{\mathrm{H}} \mathrm{H}$ & HㅌD & D $\underline{H L}$ & $\mathrm{D} \underline{\mathrm{H}} \mathrm{H}$ & $\mathrm{D} \underline{\mathrm{HD}}$ \\
\hline $4 b$ & Figure 6a & $\mathrm{CD}_{2} \mathrm{Cl}_{2}$ & 210 & 30.3 & 21.6 & 34.5 & n.r. & n.r. & 13.6 & n.r. & n.r. \\
\hline $4 b$ & Figure $6 b$ & $\mathrm{CDF}_{2} \mathrm{Cl} / \mathrm{CDF}_{3}$ & 150 & 35.6 & 21.1 & 33.6 & 14.9 & 18.7 & 9.7 & 4.0 & 5.7 \\
\hline
\end{tabular}

Light particle wildcard $\mathrm{L}=\mathrm{H}$ or $\mathrm{D}$. Temperatures $T$ are given in Kelvin, intensities in each spectrum are normalized to a sum of 100. The assignment of isotopologues and their isotopomers follows the syntax introduced in the respective main text Figures. Entries n.d. = not determined; n.r. = not resolved. 
Table S 8. Abundance of the HD isotopomers/isotopologues of $3 \mathbf{b}$ and $3 c$.

a.

\begin{tabular}{|l|l|l|l|l|l|l|l|l|l|l|l|}
\hline species & spectrum & solvent & $T$ & \multicolumn{1}{l|}{$\mathrm{H}$ NMR } & \multicolumn{1}{l|}{$\mathrm{C}$ NMR } \\
\hline & & & & $x(\mathrm{HH})$ & $x(\mathrm{HD})$ & $x(\mathrm{DH})$ & $x(\mathrm{DD})$ & $x(\mathrm{HH})$ & $x(\mathrm{HD})$ & $x(\mathrm{DH})$ & $x(\mathrm{DD})$ \\
\hline 3b & Figure 3d & $\mathrm{CD}_{2} \mathrm{Cl}_{2}$ & 185 & 0.31 & 0.31 & 0.19 & 0.19 & 0.35 & 0.30 & 0.17 & 0.17 \\
\hline 3b & Figure 3e & $\mathrm{CDF}_{2} \mathrm{Cl} / \mathrm{CDF}_{3}$ & 130 & 0.35 & 0.35 & 0.15 & 0.15 & 0.33 & 0.40 & 0.13 & 0.15 \\
\hline 3b & Figure 3f & $\mathrm{CDF}_{2} \mathrm{Cl} / \mathrm{CDF}_{3}$ & 130 & 0.50 & 0.30 & 0.12 & 0.08 & 0.48 & 0.35 & 0.12 & 0.05 \\
\hline 3c & Figure 5 & $\mathrm{CDF}_{2} \mathrm{Cl} / \mathrm{CDF}_{3}$ & 140 & 0.65 & 0.25 & 0.073 & 0.028 & n.d. & n.d. & n.d. & n.d.
\end{tabular}

b.

\begin{tabular}{|l|l|l|l|l|l|l|l|l|l|l|l|}
\hline species & spectrum & solvent & $T$ & \multicolumn{1}{|l|}{${ }^{1} \mathrm{H}$ NMR } \\
\hline & & & & $x(\mathrm{HHH})$ & $x(\mathrm{HHD})$ & $x(\mathrm{HDH})$ & $x(\mathrm{HDD})$ & $x(\mathrm{DHH})$ & $x(\mathrm{DHD})$ & $x(\mathrm{DDH})$ & $x(\mathrm{DDD})$ \\
\hline $\mathbf{4 b}$ & Figure 6a & $\mathrm{CD}_{2} \mathrm{Cl}_{2}$ & $\mathbf{2 1 0}$ & \multicolumn{2}{|c|}{0.42} & \multicolumn{2}{|c|}{0.28} & \multicolumn{2}{|c|}{0.18} & \multicolumn{2}{|c|}{0.12} \\
\hline $\mathbf{4 b}$ & Figure 6b & $\mathrm{CDF}_{2} \mathrm{Cl} / \mathrm{CDF}_{3}$ & $\mathbf{1 5 0}$ & 0.21 & 0.27 & 0.13 & 0.16 & 0.06 & 0.08 & 0.04 & 0.05
\end{tabular}

Light particle wildcard $\mathrm{L}=\mathrm{H}$ or D. See also Section 2.3.2 of this document. Temperatures $T$ are given in Kelvin. The assignment of isotopologues and their isotopomers follows the conventions in the respective main text figures. Entries n.d. $=$ not determined. In sub-table b, joined cell entries correspond to sums of abundances e.g. $x(\mathrm{HHH})+$ $x(\mathrm{HHD})=x(\mathrm{HHL})=0.42$.

Table S 9. Deuterium fractions in mobile proton sites and isotope fractionation in $3 b, 3 c$ and $4 b$.

\begin{tabular}{|l|l|l|l|l|l|l|l|l|l|l|l|l|}
\hline species & spectrum & solvent & $T$ & \multicolumn{1}{l|}{$\mathrm{H}$ NMR } & \multicolumn{3}{l|}{${ }^{13} \mathrm{C}$ NMR } \\
\hline & & & & $x(\underline{\mathrm{D}})$ & $x(\mathrm{~L} \underline{\mathrm{D}})$ & $x(\mathrm{~L} \underline{\mathrm{D}}) / x(\underline{\mathrm{D}})$ & $x(\mathrm{D})$ & $x(\underline{\mathrm{DL}})$ & $x(\underline{\mathrm{L}})$ & $x(\underline{\mathrm{L}}) / x(\underline{\mathrm{D}})$ & $x(\mathrm{D})$ \\
\hline 3b & Figure 3d & $\mathrm{CD}_{2} \mathrm{Cl}_{2}$ & 185 & 0.38 & 0.50 & 1.3 & 0.44 & 0.35 & 0.48 & 1.4 & & 0.41 \\
\hline 3b & Figure 3e & $\mathrm{CDF}_{2} \mathrm{Cl} / \mathrm{CDF}_{3}$ & 130 & 0.29 & 0.51 & 1.8 & 0.40 & 0.28 & 0.54 & 2.0 & & 0.41 \\
\hline 3b & Figure 3f & $\mathrm{CDF}_{2} \mathrm{Cl} / \mathrm{CDF}_{3}$ & 130 & 0.20 & 0.38 & 1.9 & 0.29 & 0.17 & 0.40 & 2.4 & & 0.29 \\
\hline 3c & Figure 5 & $\mathrm{CDF}_{2} \mathrm{Cl} / \mathrm{CDF}_{3}$ & 140 & 0.1 & 0.28 & 2.8 & 0.19 & n.d. & n.d. & n.d. & n.d.
\end{tabular}

b.

\begin{tabular}{|l|l|l|l|l|l|l|l|l|l|}
\hline species & spectrum & solvent & $T$ & \multicolumn{5}{|l|}{$\mathrm{H}$ NMR } \\
\hline & & & & $x(\underline{\mathrm{DLL}})$ & $x(\mathrm{~L} \underline{\mathrm{DL}})$ & $x(\mathrm{LL} \underline{\mathrm{D}})$ & $x(\mathrm{D})$ & $x(\underline{\mathrm{LL}}) / x(\mathrm{DLL})$ & $x(\mathrm{LLD}) / x(\mathrm{LDL})$ \\
\hline $\mathbf{4 b}$ & Figure 6a & $\mathrm{CD}_{2} \mathrm{Cl}_{2}$ & 210 & 0.28 & 0.42 & n.d. & n.d. & n.d. & 1.5 \\
\hline $\mathbf{4} \mathbf{b}$ & Figure 6b & $\mathrm{CDF}_{2} \mathrm{Cl} / \mathrm{CDF}_{3}$ & 150 & $\mathbf{0 . 2 2}$ & 0.37 & 0.56 & 0.39 & $\mathbf{2 . 5}$ & 1.7
\end{tabular}

Light particle wildcard $\mathrm{L}=\mathrm{H}$ or D. See also Section 2.3.2 of this document. Temperatures $T$ are given in Kelvin. The assignment of isotopologues and their isotopomers follows the conventions introduced in respective Figures, i.e. $x(\underline{D} \mathrm{~L})$ is the deuterium fraction in the H-bond of the carboxylic group; $x(\mathrm{D})$ is the all-over deuterium fraction. Entries n.d. $=$ not determined. 
Table S 10. Spectroscopic parameters of the $\pi-\pi^{*}$ absorption bands of 4-nitrophenols, their tetraethylammonium salts and homoconjugated anions in $\mathrm{CD}_{2} \mathrm{Cl}_{2}$ solution at $170-180 \mathrm{~K}$.

\begin{tabular}{|l|l|l|l|l|l|l|l|l|l|l|l|}
\hline & \multicolumn{3}{|c|}{ neutral form } & \multicolumn{3}{l|}{$\begin{array}{l}\text { tetraethylammonium } \\
\text { phenolates }\end{array}$} & \multicolumn{3}{l|}{$\begin{array}{l}\text { tetraethylammonium } \\
\text { hydrogenbisphenolates }\end{array}$} \\
\hline & $\lambda_{\max }$ & $\tilde{v}_{C O G}$ & $\lambda_{\text {COG }}$ & $\Delta \widetilde{v}$ & $\lambda_{\max }$ & $\tilde{v}_{C O G}$ & $\lambda_{\text {COG }}$ & $\Delta \widetilde{v}$ & $\lambda_{\max }$ & $\tilde{v}_{C O G}$ & $\Delta \widetilde{v}$ \\
\hline $\begin{array}{l}\text { 2,5-dichloro-4- } \\
\text { nitrophenol }\end{array}$ & 298 & 33480 & 299 & 7620 & 420 & 24460 & 409 & 2990 & 383 & 28550 & 5320 \\
\hline $\begin{array}{l}\text { 2-chloro-4- } \\
\text { nitrophenol }\end{array}$ & 310 & 33220 & 299 & 6000 & 433 & 23540 & 425 & 2660 & 390 & 27360 & 5410 \\
\hline 4-nitrophenol & 314 & 32860 & 304 & 5990 & 431 & 23790 & 420 & 2820 & 395 & 27270 & 5129 \\
\hline $\begin{array}{l}\text { 2-tert-butyl-4- } \\
\text { nitrophenol }\end{array}$ & 325 & 31690 & 316 & 5470 & 445 & 23040 & 434 & 2680 & 412 & 26220 & 5090 \\
\hline $\begin{array}{l}\text { 2,6-dimethyl-4- } \\
\text { nitrophenol }\end{array}$ & 325 & 31820 & 314 & 5490 & 453 & 22650 & 442 & 2720 & & 25950 & \\
\hline
\end{tabular}

Parameters are center of gravity $\tilde{v}_{\mathrm{COG}}$, half height full width $\Delta \widetilde{v}$ and wavelength of maximum in absorbance $\lambda_{\text {max }}$. Wavenumbers $\tilde{v}$ are given in $\mathrm{cm}^{-1}$ and wavelengths $\lambda$ in $\mathrm{nm}$. 


\section{References}

(1) Gardner, P. D.; Rafsanjani, H. S.; Rand, L. J. Am. Chem. Soc. 1959, 81, 3364-3367.

(2) Arct, J.; Hetmanek, J. Org. Prep. Proced. Int. 1976, 8, 267-270.

(3) Bew, S. P.; Hughes, D. L., Sharma, S. V. J. Org. Chem. 20o6, 71, 7881-7884.

(4) Clinton, C. F.; Aldrichimica Acta 1974, 7(1), 7-8.

(5) Siegel, J.S.; Anet, F.A.I. J. Org. Chem. 1988, 53, 2629.

(6) Carmen Carreiio, M., Garcia Ruano, J, L., Sanz, G., Toledo, M.A., Urbano, A., J. Org. Chem. 1995, 6o, 5328-5331.

(7) Mertink, F. Diploma Thesis, Freie Universität Berlin 2007.

(8) It was observed that the Mannich bases tend to be considerably less soluble than the reactants. The problem of double substitution could be kept to a minimum if conditions were found under which major fractions of the product precipitated from solution during the progress of the reaction.

(9) Van De Weter, R. W.; Pettus, T. R. R. Tetrahedron 2002, 58, 5367-5405.

(10) Cook, F. L.; Bowers, C. W.; Liotta, C. L. J. Org. Chem. 1974, 39, 3416-3418.

(11) Clinton, C. F.; Aldrichimica Acta 1974, 7(1), 7-8.

(12) (a) Nagta, W.; Okada, Kyo; Aoki, T. Synthesis 1979, 365-368. (b) Casiraghi, G.; Casnati, G.; Puglia, G.; Sartori, G. Synthesis 1980, 124-125.

(13) Suzuki, Yuji; Takahashi, Hiroshi; Chem. Pharm. Bull. 1983, 31, 1751-1753.

(14) Arenz, C.; Giannis, A. Eur. J. Org. Chem. 2001; 137-140.

(15) Johannissen, L. O.; Irebo, T.; Sjodin, M.; Johansson, O.; Hammarstrom, L. J. Phys. Chem. B 2009, 113, 16214-16225.

(16) Quantitative reaction of the substrate is crucial because its separation from the product is problematic. Progress of the reaction in terms of residual reagent can be monitored by the ${ }^{1} \mathrm{H}$ NMR signals of methylene groups in $\mathrm{N}$-chlorosuccinimide $\left(2.90 \mathrm{ppm}\right.$ in $\mathrm{CDCl}_{3}$ solution) in distinction of those in the reaction product succinimide (2.75 ppm).

(17) The ${ }^{1} \mathrm{H}$ NMR spectrum gives evidence for the presence of a quaternary ammonium. It remains unclear whether there was a zwitterionic molecule in a mixture with sodium iodide or an organic double salt (sodium phenolate/ammonium iodide).

(18) Chen, H.; Boiziau, J.; Parker, F.; Mailliet, P.; Commercon, A. J. Med. Chem. 1994, 37, 845-859.

(19) For the effective alkylation of the Mannich base approx. one equivalent of potassium hydroxide had been added; the product should thus be a mixed potassium iodide salt. The maximum amount of the organic component corresponds to the amount of Mannich base originally employed in the alkylation.

(20) (a) Tolstoy, P. M.; Koeppe, B.; Denisov, G. S.; Limbach, H. H. Angew. Chem. Int. Ed. 2oog, 48, 5745-5747; (b) Koeppe, B.; Tolstoy, P. M.; Limbach H.- H. J. Am. Chem. Soc. 2011, 133, 7897-7908.

(21) Koeppe, B.; Guo, J.; Tolstoy, P. M.; Denisov, G. S.; Limbach, H.-H J. Am. Chem. Soc. 2o13, 135, 7553-7566. 